MPP-2006-16

LMU-ASC 11/06

hep-th/0603015

\title{
Heterotic GUT and Standard Model Vacua from simply connected Calabi-Yau Manifolds
}

\author{
Ralph Blumenhagen ${ }^{\curvearrowright}$, Sebastian Moster $^{\curvearrowright}$ and Timo Weigand ${ }^{\odot,}$ \\ $\odot$ Max-Planck-Institut für Physik, Föhringer Ring 6, \\ 80805 München, Germany \\ - Arnold-Sommerfeld-Center for Theoretical Physics, Department für Physik, \\ Ludwig-Maximilians-Universität München, Theresienstraße 37, 80333 München, \\ Germany \\ blumenha,moster, weigand @mppmu.mpg.de
}

\begin{abstract}
We consider four-dimensional supersymmetric compactifications of the $E_{8} \times$ $E_{8}$ heterotic string on Calabi-Yau manifolds endowed with vector bundles with structure group $S U(N) \times U(1)$ and five-branes. After evaluating the Green-Schwarz mechanism and deriving the generalized DonaldsonUhlenbeck-Yau condition including the five-brane moduli, we show that this construction can give rise to GUT models containing $U(1)$ factors like flipped $S U(5)$ or directly the Standard Model even on simply connected Calabi-Yau manifolds. Concrete realizations of three-generation models on elliptically fibered Calabi-Yau manifolds are presented. They exhibit the most attractive features of flipped $S U(5)$ models such as doublet-triplet splitting and proton stability. In contrast to conventional GUT string models, the tree level relations among the Standard Model gauge couplings at the GUT scale are changed.
\end{abstract}




\section{Contents}

1 Introduction and Summary 2

2 The $E_{8} \times E_{8}$ heterotic string with $U(N)$ bundles and five-branes 6

2.1 Review of model building constraints . . . . . . . . . . 6

2.2 Green-Schwarz mechanism with M5-branes . . . . . . . . . . . . 8

2.3 M-theorv origin of new GS-terms . . . . . . . . . . . . . . 11

2.4 Gauge-axion masses . . . . . . . . . . . . . . . . . . . . . . . 14

2.5 Gauge couplings. . . . . . . . . . . . . . . . . . . . . . 15

2.6 Favet-Iliopoulos terms $\ldots \ldots \ldots \ldots$

2.7 D-term potential for M5-branes . . . . . . . . . . . 20

$3 \quad$ Flipped $S U(5) \times U(1)_{X} \quad 21$

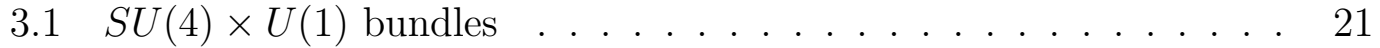

3.2 Yukawa couplings and proton decav . . . . . . . . . . . . 25

4 Three-generation models 26

4.1 Ellipticallv fibered Calabi-Yau manifolds . . . . . . . . . . . 26

4.2 Spectral cover construction . . . . . . . . . . . . . . . . 27

4.3 An example on $\mathrm{dP}_{\mathrm{A}} \ldots \ldots \ldots \ldots \ldots$

5 Just the $S U(3) \times S U(2) \times U(1)_{Y}$ gauge symmetry 33

$5.1 \quad S U(5) \times U(1)$ bundles . . . . . . . . . . . . . . . . . . 33

5.2 Gauge coupling unification . . . . . . . . . . . . . . . . . 35

6 Conclusions 36

A Normalization of the Green-Schwarz term from Type I string theory 38

\begin{tabular}{ll}
\hline B Three-generation examples & 40
\end{tabular}

\section{Introduction and Summary}

If nature really knows about superstring theory, it must of course be possible to embed the observed Standard Model of particle physics into the theory. Most attempts to verify this hope explicitly and thereby provide the first ultra-violet completion of the low-energy effective quantum field theory initially focused on the $E_{8} \times E_{8}$ heterotic corner of the M-theory star. The attitude has been to break the original ten-dimensional gauge group down to phenomenologically appealing GUT groups and to recover the observed light matter as irreducible representations of the latter [1]. The classic approaches comprise compactifications on 
toroidal orbifolds or more generally on Calabi-Yau manifolds endowed with stable holomorphic vector bundles. In the latter case, giving VEVs to the internal field strengths with values in, say, an $S U(4)$ or $S U(5)$ subgroup of one of the $E_{8}$ factors leads to the respective commutant in $E_{8}, S O(10)$ or $S U(5)$ as the fourdimensional gauge group. The $\mathbf{2 4 8}$ representation of $E_{8}$ very naturally splits into the respective GUT multiplets which incorporate the chiral fermions of the Standard Model. However, in this construction it is impossible to realize the GUT breaking further down to $S U(3) \times S U(2) \times U(1)_{Y}$ via a field theoretic Higgs mechanism, simply because the required vector-like pairs from which the GUT Higgs could arise are not present in the particle spectrum ${ }^{1}$. This necessitates the use of discrete Wilson lines as an alternative GUT breaking mechanism and considerably complicates the construction of heterotic Standard Model vacua. The point is that in order to have these Wilson lines at our disposal, we need to work on Calabi-Yau manifolds with non-zero first fundamental group, and constructing them explicitly takes quite some effort. It has been one of the recent triumphs of string model building to provide classes of such Calabi-Yau manifolds as quotients of manifolds under an appropriate freely-acting orbifold group and to construct non-abelian vector bundles on them [2-7]. Globally defined realistic models from $S U(5) \mathrm{GUT}$ in this context have been provided in [8]. For realistic models from $S O(10)$ see $[9,10] .{ }^{2}$ A recent construction of realistic models in the setup of heterotic toroidal orbifolds can be found in [13].

Independently of the heterotic model building industry, the discovery of Dbranes has opened up a complementary path to incorporating gauge interactions into string theory, more precisely the Type II theory or orientifolds thereof. The extensive analysis of intersecting D-brane models in Type IIA (for the most recent review and references consult [14]) demonstrated that not only non-abelian, but also abelian background gauge instantons can be of phenomenological interest. The connection between the two pictures is that the objects mirror dual to D6-branes at angles are magnetized D9-branes in Type I theory, which in turn are S-dual to abelian background bundles in the $S O(32)$ heterotic theory. It is therefore of obvious relevance to explore the usually neglected use of non-trivial line bundles ${ }^{3}$ in heterotic compactifications with the hope of extending our model building possibilities beyond the classic embedding of vector bundles with vanishing first class only. Likewise, one might wonder if turning on also non-abelian gauge bundles on D9-branes in Type I might lead to interesting constructions. A step into that direction has been performed in [20-22], where it has been demon-

\footnotetext{
${ }^{1}$ Note, however, that in the context of higher-level Kac-Moody algebras GUT Higgses can be realized.

${ }^{2}$ For these latter constructions, stability of the visible sector bundle has been proven in $[11,12]$. However, as of this writing, no stable hidden bundle has been found in order to satisfy the tadpole cancellation condition. As they stand, the models are therefore not yet globally defined.

${ }^{3}$ For some early references see [15-18] and more recently [19].
} 
strated that vacua with Standard-Model like gauge group and matter do indeed exist in the framework of these non-abelian braneworlds.

Building upon and extending the results of $[21,23]$, it is the aim of this article to construct realistic vacua from the $E_{8} \times E_{8}$ string with general (non-)abelian vector bundles. We will see that the embedding of $S U(4) \times U(1)$ bundles very naturally leads to the breaking of $E_{8}$ down to the GUT group $S U(5) \times U(1)_{X}$. In fact, the quantum numbers of the resulting matter representations are precisely those of the flipped SU(5) model $[24,25]$. Previous attempts to realize this scenario from different string constructions include $[26,27]$. A priori, the $U(1)_{X}$ receives a Stueckelberg mass due to the Green-Schwarz mechanism so that we seem to be back in the classic Georgi-Glashow scenario and face the problem pointed out above that we need Wilson lines for GUT breaking. By contrast, we can alternatively embed the same line bundle also into the second $E_{8}$ in such a way that the $U(1)_{X}$ is now a linear combination of the two abelian factors and remains massless. Remarkably, due to the particular way how the MSSM quantum numbers are assigned to the various representations in flipped $S U(5)$, the GUT Higgs can now arise from a $\mathbf{1 0}-\overline{\mathbf{1 0}}$ pair present in the spectrum. We can thus circumvent the necessity of working on Calabi-Yau manifolds with $\pi_{1}\left(C Y_{3}\right) \neq 0$, thereby bringing a large number of geometric backgrounds back into the heterotic model building business. Following the same rationale, it is also possible to directly arrive at the MSSM gauge group from $E_{8} \times E_{8}$ by employing $S U(5) \times U(1)$ bundles. The resulting matter representations carry exactly the MSSM quantum numbers.

Our explicit model search has focused so far on elliptically fibered Calabi-Yau manifolds: Here we profit from the spectral cover construction as the working horse to arrive at stable holomorphic vector bundles $[28,29]$. Besides, we will see that the elliptic fibration property of the Calabi-Yau admits a very natural solution to some of the constraints arising in our construction. Concretely, in both cases we embed the bundle $W=V \oplus L^{-1}$ into the first $E_{8}$ with $V$ being a $U(4)$ or $U(5)$ bundle, respectively, and the line bundle $L^{-1}$ chosen such that $c_{1}(W)=0$. The bundle $V$ arises by first constructing a stable $S U(4)$ respectively $S U(5)$ bundle à la Friedman-Morgan-Witten and then twisting it by a line bundle [21]. Our search has provided a couple of models with precisely three chiral generations of MSSM matter and no further chiral exotics. The detailed analysis of the non-chiral part of the spectrum is of course essential and postponed to a future publication.

At the technical level, the use of non-trivial line bundles makes a very careful investigation of anomalous $U(1) \mathrm{s}$ and the associated Green-Schwarz mechanism necessary. In this respect we will extend the analysis of [23] to vacua including also heterotic five-branes. Among the new features appearing is the presence of additional Green-Schwarz counter terms which have to cancel mixed abelian anomalies due to the presence of the five-branes. The existence of these additional terms is consistent with the recent analysis of six-dimensional compactifications 
of the heterotic string with line bundles and five-branes [30]. They can be further justified by a direct derivation from heterotic M-theory. Even in the absence of abelian gauge factors, these terms contribute to the four-dimensional threshold corrections and lead to five-brane dependent expressions which have also been observed in reducing Witten's background solution to heterotic M-theory [31, 32]. Likewise, the one-loop correction of the Donaldson-Uhlenbeck-Yau equation of [23] receives five-brane dependent contributions. From the associated FayetIliopoulos terms of the effective four-dimensional supergravity theory we will find a new D-term potential for the M5-brane in heterotic M-theory induced by abelian line bundles on either of the two ten-dimensional $E_{8}$-orbifold planes.

Phenomenologically, our $S U(5) \times U(1)_{X}$ construction inherits the appealing features of the flipped $S U(5)$ scenario, in particular the natural solution of the doublet-triplet splitting problem and the automatic suppression of dangerous dimension-five operators leading to proton decay [33]. In addition, as a consequence of the particular properties of our bundle embeddings, the Standard Model Higgs turns out to carry different quantum numbers than the lepton doublet, and thus also dimension-four decay operators are absent. Since the massless $U(1)_{X}$ is actually a combination of two abelian factors from both $E_{8}$ 's, the standard GUT relation for the tree-level MSSM gauge couplings is not satisfied; however, by taking into account also the threshold corrections which manifestly depend on the Kähler moduli of the internal manifold one can achieve the usual gauge coupling unification on a codimension-one hypersurface in the Kähler moduli space. After all, we should not forget that, like in all weakly coupled heterotic constructions, the four-dimensional Planck scale comes out too low and we should really promote the model to the strong coupling Horava-Witten regime [34]. This, too, is in fact nothing else than choosing part of the Kähler moduli of the model - in this case the size of the eleventh dimension - in a phenomenologically appealing range.

The remainder of this article is organised as follows: Section 2 begins with a summary of the model building constraints for the heterotic string with line bundles. We will then outline the modifications of the Green-Schwarz mechanism in the presence of five-branes and describe the M-theory origin of the new five-brane induced counter terms. Along the way we give an independent derivation, consistent with S-duality, of the correct normalisation of the Green-Schwarz terms, which we will need for concrete model building. This computation is relegated to Appendix A. The rest of section 2 discusses the issue of gauge-axion masses, threshold corrections and one-loop corrected Fayet-Iliopoulos terms and concludes with a brief discussion of the D-term potential for five-branes generated by line bundles. In section 3 we will introduce our novel realisation of the flipped $S U(5)$ including a brief discussion of Yukawa couplings and proton decay. Section 4 contains the results of our explicit model search on a Calabi-Yau manifold elliptically fibered over $\mathrm{dP}_{4}$. We give the details of a vacuum with precisely the three-generation Standard Model chiral spectrum and no chiral exotics. Fur- 
ther such solutions which we have found are collected in Appendix B. Finally, in section 5, we describe how to directly arrive at the MSSM gauge group by a construction similar to that in section 4 . Our model search again produces a couple of realistic solutions with just the MSSM chiral spectrum, as also displayed in Appendix B. Before concluding, we comment on the issue of gauge coupling unification in our scenario.

Note added: While we were preparing the results of this project for publication, we received the two very interesting eprints [35] and [36], which also analyse aspects of heterotic vacua with abelian bundles.

\section{The $E_{8} \times E_{8}$ heterotic string with $U(N)$ bundles and five-branes}

We consider the heterotic $E_{8} \times E_{8}$ string compactified on a Calabi-Yau manifold $X$ endowed with an additional vector bundle whose structure group is embedded into the $E_{8} \times E_{8}$ ten-dimensional gauge group. In addition, we also allow for the presence of heterotic respectively M-theory five-branes.

\subsection{Review of model building constraints}

To be more concrete, in this article we investigate vector bundles of the following form

$$
W=W_{1} \oplus W_{2},
$$

where $W_{1}$ is embedded into the first $E_{8}$ and $W_{2}$ into the second. For each of these bundles we take the Whitney sum

$$
W_{i}=V_{N_{i}} \oplus \bigoplus_{m_{i}=1}^{M_{i}} L_{m_{i}}
$$

of the $U\left(N_{i}\right)$ bundle $V_{N_{i}}$ and the complex line bundles $L_{m_{i}}$. They are chosen such that

$$
c_{1}\left(W_{i}\right)=c_{1}\left(V_{N_{i}}\right)+\sum_{m_{i}=1}^{M_{i}} c_{1}\left(L_{m_{i}}\right)=0 .
$$

Consequently their structure group can be embedded into an $S U\left(N_{i}+M_{i}\right)$ subgroup of $E_{8}$. The observable gauge group, being the commutant of this structure group in $E_{8}^{(1)}$, is then given by $H=E_{\left(9-N_{i}-M_{i}\right)} \times U(1)^{M_{i}}$. Depending on the line bundles it can be further enhanced or $U(1)$ factors can become massive due to the Green-Schwarz mechanism.

Let us summarize some of the model building constraints to be satisfied [23]: 
- The vector bundles $W_{i}$ have to admit spinors, i.e.

$$
c_{1}\left(W_{i}\right) \in H^{2}(X, 2 \mathbb{Z}),
$$

which in view of (33) is automatically guaranteed for the bundles we consider.

- The Bianchi identity for the three-form $H$ implies the tadpole cancellation condition

$$
0=\frac{1}{4(2 \pi)^{2}}\left(\operatorname{tr}\left(\bar{F}_{1}^{2}\right)+\operatorname{tr}\left(\bar{F}_{2}^{2}\right)-\operatorname{tr}\left(\bar{R}^{2}\right)\right)-\sum_{a} N_{a} \bar{\gamma}_{a},
$$

to be satisfied in cohomology. Here $\bar{F}_{i}$ denotes the curvature two-form of the vector bundle $W_{i}$ and $\bar{\gamma}_{a}$ represents the four-form Poincaré dual to the irreducible internal two-cycle $\Gamma_{a}$ wrapped by the $N_{a}$ M5-branes. Note that the trace is over the fundamental representation of $E_{8}$ for the gauge bundles and over the fundamental representation of $S O(1,9)$ for the curvature twoform.

For the bundles (2) the resulting topological condition in cohomology can generally be written as

$$
\sum_{i=1}^{2}\left(\operatorname{ch}_{2}\left(V_{N_{i}}\right)+\frac{1}{2} \sum_{m_{i}=1}^{M_{i}} c_{1}^{2}\left(L_{m_{i}}\right)\right)-\sum_{a} N_{a} \bar{\gamma}_{a}=-c_{2}(T) .
$$

- At string tree level, the connection of the vector bundle has to satisfy the well-known zero-slope limit of the Hermitian Yang-Mills equations,

$$
F_{a b}=F_{\bar{a} \bar{b}}=0, \quad g^{a \bar{b}} F_{a \bar{b}}=0 .
$$

The first equation implies that each term in (2) has to be a holomorphic vector bundle. The second equation can be satisfied precisely by holomorphic $\mu$-stable bundles which meet in addition the integrability condition

$$
\int_{X} J \wedge J \wedge c_{1}\left(V_{N_{i}}\right)=0, \quad \int_{X} J \wedge J \wedge c_{1}\left(L_{m_{i}}\right)=0,
$$

for all bundles individually. We will refer to the latter constraints in the sequel as the tree-level Donaldson-Uhlenbeck-Yau (DUY) equation. As has been demonstrated in [23] for the case without any M5-branes, the DUY condition arises in the effective four-dimensional theory from a FayetIliopoulus term for the $U(1)$ gauge fields. Moreover, it receives a one-loop correction, which for the bundles we consider is of the form

$$
\begin{aligned}
& \int_{X} J \wedge J \wedge c_{1}\left(L_{m_{i}}\right)- \\
& \ell_{s}^{4} g_{s}^{2} \int_{X} c_{1}\left(L_{m_{i}}\right) \wedge\left(\operatorname{ch}_{2}\left(V_{N_{i}}\right)+\frac{1}{2} \sum_{n_{i}=1}^{M_{i}} c_{1}^{2}\left(L_{n_{i}}\right)+\frac{1}{2} c_{2}(T)\right)=0
\end{aligned}
$$


for all line bundles, where $g_{s}=e^{\phi_{10}}$ and $\ell_{s}=2 \pi \sqrt{\alpha^{\prime}}$. Of course the analogous condition for $V_{N_{i}}$ instead of $L_{m_{i}}$ is to be considered as well. In section 2.6 we will compute how this equation changes if M5-branes are included. In addition we have to require that the real part of the likewise loop-corrected gauge kinetic functions in the effective field theory are positive [20,37].

\subsection{Green-Schwarz mechanism with M5-branes}

The use of non-trivial line bundles makes a careful analysis of anomalous $U(1)$ factors indispensible. It turns out that if we want to allow for the presence of five-branes to more easily find solutions of the tadpole condition (6) , the associated Green-Schwarz mechanism gets further modified. Let us explain briefly how the Green-Schwarz anomaly cancellation mechanism works for the case that M5-branes are included. Along the way we will identify additional terms in the effective action which have to be present for a consistent coupling of the fivebranes to the bulk action. Recall that for five-branes in the $S O(32)$ heterotic string, there appears extra bifundamental chiral matter with respect to the unitary gauge groups and the symplectic gauge groups supported on the five-branes. The resulting extra contributions to the anomalies are cancelled by extra GreenSchwarz terms from the 5-branes [21].

For the $E_{8} \times E_{8}$ heterotic string the story must be different: In this case there does not exist chiral matter arising from the M5-branes and in four dimensions the M5-brane does not necessarily support a gauge field ${ }^{4}$.

Unless stated otherwise we will be working in string frame and using the conventions of [38]. In ten dimensions the gauge and gravitational anomalies are cancelled by the counter term $[39,40]^{5}$

$$
S_{G S}=\frac{1}{24(2 \pi)^{5} \alpha^{\prime}} \int B \wedge X_{8}
$$

where $B$ is the Kalb-Ramond two-form field and the eight-form $X_{8}$ reads, as usual,

$$
X_{8}=\frac{1}{24} \operatorname{Tr} F^{4}-\frac{1}{7200}\left(\operatorname{Tr} F^{2}\right)^{2}-\frac{1}{240}\left(\operatorname{Tr} F^{2}\right)\left(\operatorname{tr} R^{2}\right)+\frac{1}{8} \operatorname{tr} R^{4}+\frac{1}{32}\left(\operatorname{tr} R^{2}\right)^{2} .
$$

Explicitly taking care of the two $E_{8}$ factors by writing $F=F_{1}+F_{2}$ one gets

$$
\begin{aligned}
X_{8}= & \frac{1}{4}\left(\operatorname{tr} F_{1}^{2}\right)^{2}+\frac{1}{4}\left(\operatorname{tr} F_{2}^{2}\right)^{2}-\frac{1}{4}\left(\operatorname{tr} F_{1}^{2}\right)\left(\operatorname{tr} F_{2}^{2}\right)-\frac{1}{8}\left(\operatorname{tr} F_{1}^{2}+\operatorname{tr} F_{2}^{2}\right)\left(\operatorname{tr} R^{2}\right)+ \\
& \frac{1}{8} \operatorname{tr} R^{4}+\frac{1}{32}\left(\operatorname{tr} R^{2}\right)^{2} .
\end{aligned}
$$

\footnotetext{
${ }^{4}$ If the M5-brane wraps a two-cycle in the Calabi-Yau of genus $g$, then there exists a $U(1)^{g}$ gauge group in four dimensions [32].

${ }^{5}$ There exists some confusion in the literature about the correct normalization of this term. In appendix A we will present an independent derivation from the S-dual Type IIB orientifold point of view. Note in particular that this correct factor differs from the one used in $[20,21,23$, 30,37 by 2 .
} 

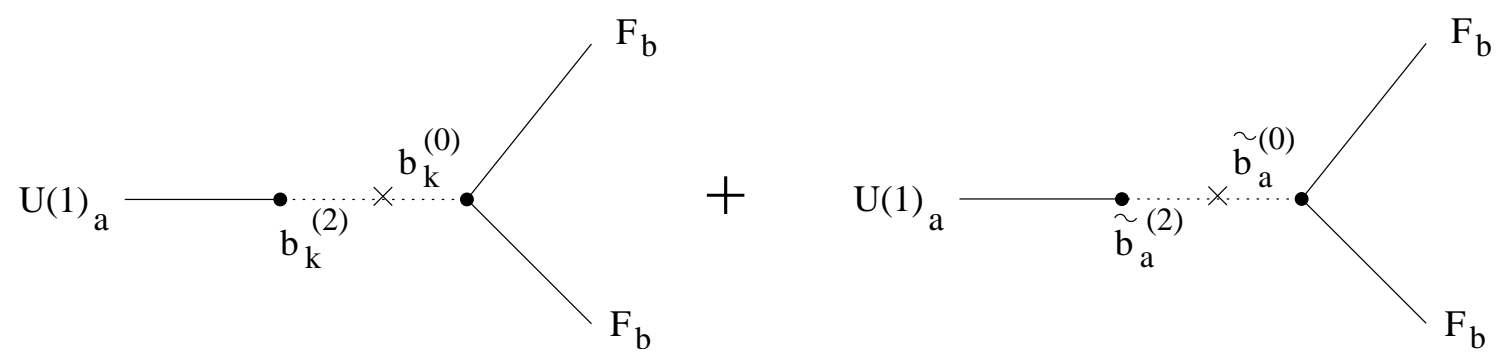

Figure 1: Green-Schwarz counter term for the mixed gauge anomaly.

Using the tadpole cancellation condition (15), we dimensionally reduce this term to

$$
\begin{aligned}
S_{G S} & =\sum_{i=1}^{2}\left\{\frac{1}{8(2 \pi)^{3} \alpha^{\prime}} \int B \wedge\left(\operatorname{tr} F_{i}^{2}\right)\left[\frac{1}{4(2 \pi)^{2}}\left(\operatorname{tr} \bar{F}_{i}^{2}-\frac{1}{2} \operatorname{tr} \bar{R}^{2}\right)-\frac{1}{3} \sum_{a} N_{a} \bar{\gamma}_{a}\right]\right. \\
& +\frac{1}{4(2 \pi)^{3} \alpha^{\prime}} \int B \wedge \operatorname{tr}\left(F_{i} \bar{F}_{i}\right)\left[\frac{1}{4(2 \pi)^{2}}\left(\operatorname{tr} \bar{F}_{i}^{2}-\frac{1}{2} \operatorname{tr} \bar{R}^{2}\right)-\frac{1}{3} \sum_{a} N_{a} \bar{\gamma}_{a}\right], \\
& \left.+\frac{1}{24(2 \pi)^{5} \alpha^{\prime}} \int B \wedge\left[\operatorname{tr}\left(F_{i} \bar{F}_{i}\right)\right]^{2}\right\} \\
& -\frac{1}{96(2 \pi)^{3} \alpha^{\prime}} \int B \wedge\left[\frac{1}{4(2 \pi)^{2}}\left(\operatorname{tr} R^{2}\right)\left(\operatorname{tr} \bar{R}^{2}\right)-2 \sum_{a} N_{a} \bar{\gamma}_{a}\right] \\
& -\frac{1}{24(2 \pi)^{5} \alpha^{\prime}} \int B \wedge \operatorname{tr}\left(F_{1} \bar{F}_{1}\right) \operatorname{tr}\left(F_{2} \bar{F}_{2}\right) .
\end{aligned}
$$

In addition one has to take into account the kinetic term

$$
S_{k i n}=-\frac{1}{4 \kappa_{10}^{2}} \int e^{-2 \phi_{10}} H \wedge \star_{10} H,
$$

where $\kappa_{10}^{2}=\frac{1}{2}(2 \pi)^{7}\left(\alpha^{\prime}\right)^{4}$ and the heterotic 3-form field strength $H=d B^{(2)}-$ $\frac{\alpha^{\prime}}{4}\left(\omega_{Y}-\omega_{L}\right)$ involves the gauge and gravitational Chern-Simons terms. Following the steps detailed in [23] and computing the diagrams as shown in figure 1 results in the Green-Schwarz six-form

$$
\begin{array}{r}
A \sim A_{\text {pert }}-\frac{1}{24(2 \pi)^{4} \alpha^{\prime}} \sum_{a} N_{a} \int_{\Gamma_{a}} \operatorname{tr}\left(F_{1} \bar{F}_{1}\right)\left[\frac{1}{4}\left(\operatorname{tr} F_{1}^{2}+\operatorname{tr} F_{2}^{2}-\operatorname{tr} R^{2}\right)+\right. \\
\left.\frac{3}{4}\left(\operatorname{tr} F_{1}^{2}-\operatorname{tr} F_{2}^{2}\right)\right]+(1 \leftrightarrow 2) .
\end{array}
$$

The term $A_{\text {pert }}$ precisely cancels the mixed abelian-nonabelian, mixed abeliangravitational and mixed abelian anomalies arising from the two $E_{8}$ factors [23]. 
Since there does not exist any chiral matter from the M5-branes, the only way to compensate the second term in (16) is by additional Green-Schwarz terms from the M5-branes. In fact, it is known that on the six-dimensional world-volume of an M5-brane there lives a self-dual tensor field $\widetilde{B}_{a}$, which by dimensional reduction gives rise to a two-form and a dual scalar. These can generate additional GreenSchwarz counter terms.

More precisely, one can show that the following extra terms

$$
S_{G S}^{(1)}=\frac{1}{96(2 \pi)^{3} \alpha^{\prime}} \sum_{a} N_{a} \int_{\Gamma_{a}} B \wedge\left(\operatorname{tr} F_{1}^{2}+\operatorname{tr} F_{2}^{2}-\operatorname{tr} R^{2}\right)
$$

and

$$
S_{G S}^{(2)}=\frac{1}{8(2 \pi)^{3} \alpha^{\prime}} \sum_{a} N_{a} \int_{\Gamma_{a}} \widetilde{B}_{a} \wedge\left(\operatorname{tr} F_{1}^{2}-\operatorname{tr} F_{2}^{2}\right)
$$

provide just the right counter terms to cancel the five-brane dependent part in (16). We would therefore like to argue that these terms have indeed to be present in the ten-dimensional effective action of the $E_{8}$ heterotic string for a consistent five-brane coupling. Even though the requirement of these terms by anomaly cancellation is manifest only once we allow for background bundles with non-zero first Chern class, their presence cannot depend on the gauge instanton background, of course. In particular, they have an effect on the gauge kinetic function also of the field strength associated with the semi-simple part of the gauge group, as we will see in section 2.5. Note that the first term (17) involves the ten-dimensional two-form $B$ and the second term (18) all the tensor fields $\widetilde{B}_{a}$ living on the M5-branes. The terms (18) can be viewed as being due to the cross terms in the kinetic action for the three-forms $\widetilde{H}_{a}{ }^{6}$

$$
S_{k i n}=-\frac{1}{2(2 \pi)^{3}\left(\alpha^{\prime}\right)^{2}} \int_{\Gamma_{a}} e^{-2 \phi} \widetilde{H}_{a} \wedge \star_{6} \widetilde{H}_{a}
$$

with

$$
\widetilde{H}_{a}=d \widetilde{B}_{a}-\frac{\alpha^{\prime}}{8}\left(\omega_{Y, 1}-\omega_{Y, 2}\right)
$$

Both contributions to the effective action are also consistent with the analogous Green-Schwarz mechanism in six-dimensional compactifications, as analysed recently in [30].

\footnotetext{
${ }^{6}$ The following normalization of $\widetilde{H}_{a}$ arises after straightforward dimensional reduction of corresponding ten-dimensional terms to six dimensions. Due to the self-duality of $\widetilde{H}_{a}$ we should actually stick to the M-theory five-brane action [41], as will be done in section 2.3., but the resulting change of normalisation of the kinetic term is just a matter of field redefinitions.
} 


\subsection{M-theory origin of new GS-terms}

The presence of these latter two counter terms (17) and (18) can also be derived directly from Horava-Witten theory. The logic is very similar to that leading to the usual Green-Schwarz terms from heterotic M-theory, as first described in $[31,42]$. Here we will extend the analysis to the five-brane dependent terms. Our derivation was done independently from [43], where a similar analysis has been performed ${ }^{7}$.

To fix our conventions, the effective action of heterotic M-theory in the upstairs picture is given by [44-46]

$$
\begin{aligned}
S & =S_{k i n}+S_{C S}+S_{\text {curv }}+S_{Y M}+S_{M 5}, \\
S_{\text {kin }} & =\frac{1}{2 \bar{\kappa}^{2}} \int_{\mathcal{M}_{u}^{11}} R \Omega-\frac{1}{2} G \wedge \star G, \\
S_{C S} & =\frac{1}{2 \bar{\kappa}^{2}} \int_{\mathcal{M}_{u}^{11}} \frac{1}{6} C \wedge G \wedge G, \\
S_{\text {curv }} & =\frac{1}{48(2 \pi)^{3} \bar{\kappa}^{2} T_{5}} \int_{\mathcal{M}_{u}^{11}} C \wedge\left(\frac{1}{8} \operatorname{tr} R^{4}-\frac{1}{32}\left(\operatorname{tr} R^{2}\right)^{2}\right), \\
S_{Y M} & =-\sum_{i=1}^{2} \frac{1}{2 \lambda^{2}} \int_{\mathcal{M}^{(10)}} \operatorname{tr}\left(F^{i} \wedge \star F^{i}\right)-\frac{1}{2} \operatorname{tr}(R \wedge \star R),
\end{aligned}
$$

where $\mathcal{M}_{u}^{11}=\mathcal{M}^{10} \times S^{1}$. The part of M5-brane action $S_{M 5}$ [41] relevant for our purposes will be given at the end of this section. The compact eleventh dimension takes values in the range $-\pi \rho<x^{11} \leq \pi \rho$ and the gauge fields are localized at $x^{11}=0, \pi \rho$. It is understood that only the field components invariant under the orbifold action $x^{11} \mapsto-x^{11}$ are present in (21) [44]. Eleven-dimensional indices will be denoted by $I, J, K, \ldots$ and ten-dimensional ones by $A, B, C, \ldots$ The tendimensional gauge couplings are related to $\bar{\kappa}$ via $\lambda^{2}=(4 \pi)\left(2 \pi \bar{\kappa}^{2}\right)^{2 / 3}$ and the tension of the five-brane is given by $T_{5}=\left(\frac{2 \pi}{\bar{\kappa}^{4}}\right)^{1 / 3}[46]$.

In the presence of five-branes, the Bianchi identity for the field strength $\mathrm{G}=d C$ gets modified as $[32]$

$(d G)_{11 A B C D}=-\frac{\bar{\kappa}^{2}}{\lambda^{2}}\left(J_{1} \delta\left(x^{11}\right)+J_{2} \delta\left(x^{11}-\pi \rho\right)+\frac{1}{2} J_{5}\left(\delta\left(x^{11}-y\right)+\delta\left(x^{11}+y\right)\right)\right)_{11 A B C D}$,

taking into account the contribution from the five-brane at $x^{11}=y$ and its mirror brane at $x^{11}=-y$. The generalisation to the case of several five-branes is obvious. The gauge and curvature sources at the orbifold fixed planes are given by $J_{i}=\operatorname{tr} F^{i} \wedge F^{i}-\frac{1}{2} \operatorname{tr} R \wedge R=d \omega_{i}$ for $i=1,2$, while the five-branes contribute

\footnotetext{
${ }^{7}$ Note that this reference does not use the resulting Green-Schwarz terms for cancellation of abelian anomalies and does not consider the terms (18) arising from the M5-brane action. Also, to the best of our knowledge, the connection between the new GS terms and the FI-D-terms in section 2.6 has not been explored previously.
} 
$J_{5}=-4(2 \pi)^{2} \delta(\Gamma)$. Here $\delta(\Gamma)$ is the four-form Poincaré dual to the worldvolume of the five-brane in $\mathcal{M}^{(10)}{ }^{8}$ In analogy with the Yang-Mills and Lorentz Chern-Simons forms we also introduce the ten-dimensional three-form $\omega_{5}$ satisfying $J_{5}=d \omega_{5}$.

Being interested in the ten-dimensional theory after Kaluza-Klein reduction on $S^{1}$, we now focus on the situation where the eleventh dimension is much smaller than the ten-dimensional space. This is the limit in which the effective action of the ten-dimensional weakly coupled heterotic string arises [31,42]. In this regime ten-dimensional derivatives of gauge and curvature terms can be neglected as compared to field variations along $x^{11}$. Hence, one can give an approximate solution for $G$ and $C$ to the above Bianchi identity and the equations of motion $D^{I} G_{I J K L}=0$ by splitting the fields into their zero-mode and a background part as $C=C^{(0)}+C^{(1)}$ and $G=G^{(0)}+G^{(1)}$. Including also the five-brane sources, we get

$$
\begin{gathered}
C_{A B C}=C_{A B C}^{(1)}, \quad C_{A B 11}=B_{A B}, \\
G_{A B C D}=G_{A B C D}^{(1)}, \quad G_{A B C 11}=(d B)_{A B C}+G_{A B C 11}^{(1)}, \\
C_{A B C}^{(1)}=-\frac{\bar{\kappa}^{2}}{2 \lambda^{2}}\left(\omega_{1} \epsilon\left(x^{11}\right)+\frac{1}{2} \omega_{5}\left(\epsilon\left(x^{11}-y\right)+\epsilon\left(x^{11}+y\right)\right)-\frac{x^{11}}{\pi \rho}\left(\omega_{1}+\omega_{2}+\omega_{5}\right)\right)_{A B C} \\
G_{A B C D}^{(1)}=-\frac{\bar{\kappa}^{2}}{2 \lambda^{2}}\left(J_{1} \epsilon\left(x^{11}\right)+\frac{1}{2} J_{5}\left(\epsilon\left(x^{11}-y\right)+\epsilon\left(x^{11}+y\right)\right)-\frac{x^{11}}{\pi \rho}\left(J_{1}+J_{2}+J_{5}\right)\right)_{A B C D} \\
G_{A B C 11}^{(1)}=-\frac{\bar{\kappa}^{2}}{2 \lambda^{2} \pi \rho}\left(\omega_{1}+\omega_{2}+\omega_{5}\right)_{A B C} .
\end{gathered}
$$

$\epsilon\left(x^{11}\right)$ denotes the step function, i.e. $\epsilon\left(x^{11}\right)=+1$ for $x^{11}$ positive and -1 otherwise. Note that the part of $G_{A B C D}^{(1)}$ linear in $x^{11}$ is cancelled precisely by the contribution from $G_{A B C 11}^{(1)}$ when the Bianchi identity is evaluated. The equations of motion for the field strength $G$ are only satisfied up to terms proportional to $\partial J_{i}$, which are assumed to be negligible in the limit we are considering $[31,42]$.

The ten-dimensional weakly coupled heterotic string theory is recovered by compactification on $S^{1}$ according to the standard ansatz

$$
d s_{11}^{2}=e^{-2 \phi / 3} g_{A B}^{(10)} d x^{A} d x^{B}+e^{(4 \phi / 3)}\left(d x^{11}\right)^{2} .
$$

In particular, the kinetic term for $G$ contains a part involving the combination $G_{11 A B C} G^{11 A B C}$. Inserting the solution (22), integrating over $S^{1}$ and focussing only on terms not involving $\omega_{5}$ due to the five-branes precisely yields the familiar kinetic term

$$
S_{\text {kin }}^{H}=-\frac{1}{4 \kappa_{10}^{2}} \int_{\mathcal{M}^{(10)}} e^{-2 \phi} H \wedge \star H
$$

${ }^{8}$ When we further compactify $\mathcal{M}^{(10)}=\mathcal{M}^{(4)} \times C Y_{3}$ we have the obvious decomposition $\delta(\Gamma)=\delta\left(\mathcal{M}^{(4)}\right) \wedge \bar{\gamma}$ for a five-brane wrapping the two-cycle dual to the four-form $\bar{\gamma}$ on $C Y_{3}$. 
for the ten-dimensional three-form field strength $H=d B-\frac{\alpha^{\prime}}{4}\left(\omega_{1}+\omega_{2}\right)$ after setting

$$
\frac{1}{\kappa_{10}^{2}}=\frac{2 \pi \rho}{\bar{\kappa}^{2}}, \quad \alpha^{\prime}=\frac{4 \bar{\kappa}^{2}}{2 \lambda^{2} \pi \rho}=\frac{2^{-1 / 3}}{\pi^{2} \rho}\left(\frac{\bar{\kappa}}{4 \pi}\right)^{2 / 3} .
$$

We are now ready to investigate the origin of the complete Green-Schwarz counter terms including the contribution from the five-branes. They arise at order $\left(\frac{\bar{\kappa}^{2}}{2 \lambda^{2}}\right)^{2}$ after inserting the above solution for $C$ and $G$ into the Chern-Simons terms $S_{C S}$ in (21) as

$$
\begin{aligned}
\left.S_{C S}\right|_{\left(\frac{\bar{\kappa}^{2}}{2 \lambda^{2}}\right)^{2}} & =\frac{3}{12 \bar{\kappa}^{2}} \int_{\mathcal{M}^{(10)}} \int_{S^{1}} B \wedge G^{(1)} \wedge G^{(1)} \wedge d x^{11} \\
& =\frac{\pi \rho}{4 \bar{\kappa}^{2}}\left(\frac{\bar{\kappa}^{2}}{2 \lambda^{2}}\right)^{2} \int_{\mathcal{M}^{(10)}} B \wedge\left(\frac{2}{3}\left(J_{1}^{2}+J_{2}^{2}-J_{1} J_{2}\right)-\frac{1}{6} J_{5}\left(J_{1}+J_{2}\right)\right)
\end{aligned}
$$

plus additional terms proportional to $\int B \wedge J_{5}^{2}$, which however vanish after performing the integral. To arrive at this expression we place the five-brane and its mirror symmetrically at $y= \pm \frac{\pi \rho}{2}$ between the two orbifold fixed-planes. Additional contributions from the higher curvature corrections $S_{\text {curv }}$ are

$$
\begin{aligned}
S_{\text {curv }} & =\frac{1}{48(2 \pi)^{3} \bar{\kappa}^{2} T_{5}} \int_{\mathcal{M}_{u}^{11}} C \wedge\left(\frac{1}{8} \operatorname{tr} R^{4}-\frac{1}{32}\left(\operatorname{tr} R^{2}\right)^{2}\right) \\
& =\frac{1}{24(2 \pi)^{5} \alpha^{\prime}} \int_{\mathcal{M}^{(10)}} B \wedge\left(\frac{1}{8} \operatorname{tr} R^{4}-\frac{1}{32}\left(\operatorname{tr} R^{2}\right)^{2}\right) .
\end{aligned}
$$

The part $\frac{2}{3}\left(J_{1}^{2}+J_{2}^{2}-J_{1} J_{2}\right)$ in (26) combines with (27) into the standard Green-Schwarz eight-form $X_{8}[31,42]$.

The additional counter terms (17) we are after now arise from $J_{5}\left(J_{1}+J_{2}\right)=$ $-4(2 \pi)^{2} \delta(\Gamma) \wedge\left(\operatorname{tr} F_{1}^{2}+\operatorname{tr} F_{2}^{2}-\operatorname{tr} R^{2}\right)$. In summary, (26) and (27) yield in the ten-dimensional limit

$$
S_{G S}=c \int_{\mathcal{M}^{(10)}} B \wedge\left(X_{8}+\frac{(2 \pi)^{2}}{4} \delta(\Gamma) \wedge\left(\operatorname{tr} F_{1}^{2}+\operatorname{tr} F_{2}^{2}-\operatorname{tr} R^{2}\right)\right)
$$

with

$$
c=\frac{8}{3} \frac{\pi \rho}{4 \bar{\kappa}^{2}}\left(\frac{\bar{\kappa}^{2}}{2 \lambda^{2}}\right)^{2}=\frac{1}{24(2 \pi)^{5} \alpha^{\prime}},
$$

as postulated in (17).

The origin of the second five-brane dependent counter term (18) lies in the M5-brane action. With the normalisations of [41] (see e.g. also [47]), the part relevant for our analysis is given by

$$
S_{M 5}=-\frac{T_{5}}{2} \sum_{a} N_{a} \int_{\Gamma_{a} \cup \Gamma_{a}^{\prime}}\left(\frac{1}{4} \widetilde{F}_{a} \wedge \star \widetilde{F_{a}}+\tilde{C}+\frac{1}{2} d \widetilde{B}_{a} \wedge C\right),
$$


again summing over all branes and their mirrors. Here $\widetilde{F}_{a}=d \tilde{B}_{a}-C$ is the modified field strength of the self-dual tensor field $\tilde{B}_{a}$ living on the five-brane and $\tilde{C}$ is the bulk six-form potential dual to $C$. The contribution from (30) we are interested in is the topological coupling $d \tilde{B}_{a} \wedge C$. Following the general strategy we insert again the appropriate background solution for $C$ and place brane and mirror brane at $y= \pm \frac{\pi \rho}{2}$ respectively to find

$$
\begin{aligned}
S_{\text {top }} & =-\frac{T_{5}}{4} \sum_{a} N_{a}\left(\int_{\Gamma_{a}} \widetilde{B}_{a} \wedge d C^{(1)}+\int_{\Gamma_{a}^{\prime}} \widetilde{B}_{a} \wedge d C^{(1)}\right)= \\
& =\frac{T_{5}}{4} \frac{\bar{\kappa}^{2}}{2 \lambda^{2}} \sum_{a} N_{a} \int_{\Gamma_{a}} \widetilde{B}_{a} \wedge\left(\operatorname{tr} F_{1}^{2}-\operatorname{tr} F_{2}^{2}\right) .
\end{aligned}
$$

It can be checked that, together with the kinetic term for $\widetilde{B}_{a}$, this coupling indeed yields precisely the required counter terms to cancel the contribution to the five-brane anomaly in the second line of (16). In the standard ten-dimensional normalisation of the kinetic action for $\widetilde{B}_{a}$ which we used in (19) one eventually recovers the counter term (18). Note that we are always free to change the normalization of $\widetilde{B}_{a}$; different normalizations simply correspond to different physical meanings of the modulus $\lambda_{a}$, to be introduced in section 2.5., which is associated to the position of the five-brane along $S^{1}$.

\subsection{Gauge-axion masses}

After this M-theoretic interlude we turn our attention to some relevant aspects of the four-dimensional effective action of our upcoming construction of flipped $S U(5) \times U(1)_{X}$ and $S U(3) \times S U(2) \times U(1)_{Y}$ vacua. A central question we need to address is whether the abelian gauge factors really remain massless after the Green-Schwarz mechanism cancels potential anomalies. For this purpose, we summarize in this section the various axion-gauge boson mass terms.

In the presence of five-branes, three kinds of axions come into the play: the universal axion, $b_{0}^{(0)}$, the Kähler-axions, $b_{k}^{(0)}$, and finally the five-brane axions $\widetilde{b}_{a}^{(0)}$.

They arise, together with their Hodge dual counterparts, from the reduction of the Kalb-Ramond form, its dual six-form and the self-dual two-forms on the five-branes

$$
\begin{aligned}
B^{(2)} & =b_{0}^{(2)}+\ell_{s}^{2} \sum_{k=1}^{h_{11}} b_{k}^{(0)} \omega_{k}, \quad B^{(6)}=\ell_{s}^{6} b_{0}^{(0)} \operatorname{vol}_{6}+\ell_{s}^{4} \sum_{k=1}^{h_{11}} b_{k}^{(2)} \widehat{\omega}_{k}, \\
\widetilde{B}_{a} & =\widetilde{b}_{a}^{(2)}+\ell_{s}^{2} \widetilde{b}_{a}^{(0)} \widehat{\gamma}_{a} .
\end{aligned}
$$

Here we have introduced a basis $\omega_{k},\left(k=1, \ldots, h_{11}\right)$ of $H^{2}(X, \mathbb{Z})$ together with the Hodge dual four-forms $\widehat{\omega}_{k}$ normalised such that $\int_{X} \omega_{k} \wedge \widehat{\omega}_{k^{\prime}}=\delta_{k, k^{\prime}}$. 
Likewise $\widehat{\gamma}_{a}$ is the Hodge dual of $\bar{\gamma}_{a}$. Furthermore vol $_{6}$ represents the volume form of the internal Calabi-Yau manifold normalised such that $\int_{X} \operatorname{vol}_{6}=1$.

The final piece of notation we will need occurs in expressing relevant traces as

$$
\begin{aligned}
\operatorname{tr}_{E_{8}^{(i)}}\left(F_{i} \bar{F}_{i}\right) & =\sum_{m_{i}, n_{i}=1}^{M_{i}} \kappa_{m_{i}, n_{i}} f_{m_{i}} \bar{f}_{n_{i}} \\
\operatorname{tr}_{E_{8}^{(i)}}\left(F_{i}^{2}\right) & =2 \operatorname{tr}_{E_{9-N_{i}-M_{i}}}\left(F_{i}^{2}\right)+\sum_{m_{i}, n_{i}=1}^{M_{i}} \eta_{m_{i}, n_{i}} f_{m_{i}} f_{n_{i}}
\end{aligned}
$$

where the $f_{m_{i}}$ and $\bar{f}_{m_{i}}$ denote the observable and internal $U(1)$ gauge fields. Note that the non-abelian part of the traces carries different numerical pre-factors for bundles other than the one used in this article (see e.g. [30]). It is then a straightforward task to extract the mass terms for the various axions (or equivalently their dual two-forms) from the heterotic action [23]: The mass terms for the universal axion reads

$$
\begin{aligned}
M_{0, m_{i}}= & \frac{1}{4(2 \pi)^{2} \alpha^{\prime}} \int_{\mathcal{M}^{(4)}} b_{0}^{(2)} \wedge f_{m_{i}}\left[\sum _ { n _ { i } = 1 } ^ { M _ { i } } \kappa _ { m _ { i } , n _ { i } } \int _ { X } c _ { 1 } ( L _ { n _ { i } } ) \wedge \left(\operatorname{ch}_{2}\left(V_{N_{i}}\right)+\right.\right. \\
& \left.\left.\frac{1}{2} \sum_{k_{i}=1}^{M_{i}} c_{1}^{2}\left(L_{k_{i}}\right)+\frac{1}{2} c_{2}(T)-\frac{1}{4} \sum_{a} N_{a} \bar{\gamma}_{a}\right)\right] .
\end{aligned}
$$

For the Kähler axions the kinetic term for $H_{3}$ induces the mass terms

$$
M_{k, m_{i}}=\frac{1}{2(2 \pi)^{2} \alpha^{\prime}} \int_{\mathcal{M}^{(4)}} b_{k}^{(2)} \wedge f_{m_{i}}\left[\sum_{n_{i}=1}^{M_{i}} \kappa_{m_{i}, n_{i}} \int_{X} c_{1}\left(L_{n_{i}}\right) \wedge \widehat{\omega}_{k}\right]
$$

and the 5-brane Green-Schwarz term yields the mass term

$$
M_{a, m_{i}}= \pm \frac{1}{4(2 \pi)^{2} \alpha^{\prime}} \int_{\mathcal{M}^{(4)}} \widetilde{b}_{a}^{(2)} \wedge f_{m_{i}}\left[\sum_{n_{i}=1}^{M_{i}} \kappa_{m_{i}, n_{i}} \int_{X} c_{1}\left(L_{n_{i}}\right) \wedge \bar{\gamma}_{a}\right]
$$

for the 5-brane axions. The plus sign holds for $E_{8}^{(1)}$ and the minus sign for $E_{8}^{(2)}$.

A combination of abelian gauge fields or of axions, respectively, remains massless, if it lies in the kernel of this whole axion-gauge boson mass matrix $\left(M_{0}, M_{k}, M_{a}\right)_{m_{i}}$.

\subsection{Gauge couplings}

In this section we extract the holomorphic gauge kinetic functions for the nonabelian and abelian gauge groups [40,48-51]. The tree-level gauge kinetic function 
for the non-abelian factor is still simply $f=S$ in terms of the complexified dilaton

$$
S=\frac{1}{2 \pi}\left[e^{-2 \phi_{10}} \frac{\operatorname{Vol}(X)}{\ell_{s}^{6}}+i b_{0}^{(0)}\right] .
$$

Let us furthermore define

$$
\frac{1}{4(2 \pi)^{2}} \operatorname{tr} \bar{F}_{i}^{2}=\sum_{k=1}^{h_{11}}\left(\operatorname{tr} \bar{F}_{i}^{2}\right)_{k} \widehat{\omega}_{k}, \quad \quad \bar{\gamma}_{a}=\sum_{k=1}^{h_{11}}\left(\bar{\gamma}_{a}\right)_{k} \widehat{\omega}_{k}
$$

Then the one-loop corrected gauge kinetic function for the non-abelian gauge fields in the large radius regime can be written as

$$
f_{1,2}=S+\frac{1}{8} \sum_{k=1}^{h_{11}} T_{k}\left(\operatorname{tr} \bar{F}_{1,2}^{2}-\frac{1}{2} \operatorname{tr} \bar{R}^{2}-\sum_{a} N_{a} \bar{\gamma}_{a}\right)_{k} \pm \frac{1}{2} \sum_{a} N_{a} \Lambda_{a} .
$$

The complex scalars appearing above are the bosonic part of the $\mathcal{N}=1$ superfields and given by

$$
\begin{aligned}
T_{k} & =\frac{1}{2 \pi}\left[-\frac{1}{\ell_{s}^{2}} \int_{X} J \wedge \widehat{\omega}_{k}+i b_{k}^{(0)}\right] \\
\Lambda_{a} & =\frac{1}{2 \pi}\left[-\lambda_{a} \frac{\operatorname{Vol}\left(\Gamma_{a}\right)}{\ell_{s}^{2}}+i \widetilde{b}_{a}^{(0)}\right]
\end{aligned}
$$

The $\lambda_{a}$ denote the scalars which together with the self-dual two-forms $\widetilde{B}_{a}$ combine into tensor multiplets on the six-dimensional world-volume of the five-branes. In the strong coupling Horava-Witten model these scalars are nothing else than the position of the respective five-branes along the eleventh direction.

In fact, what one can easily read off from the dimensionally reduced heterotic action are the couplings between the various axions and the gauge fields and thus the imaginary part of (39). The relevant contributions stem again partly from the Green-Schwarz terms and the cross-terms of the kinetic action for the underlying two-form fields (see again [23] for the details in our context). It is of course the power of four-dimensional $\mathcal{N}=1$ supergravity which allows us to simply reconstruct the full expression (39) since the gauge kinetic functions are holomorphic. Consequently, for the real part, i.e. for the gauge couplings, one gets at linear order in $\lambda_{a}$

$$
\begin{aligned}
\frac{4 \pi}{g_{1,2}^{2}}= & \frac{e^{-2 \phi_{10}}}{3 \ell_{s}^{6}} \int_{X} J \wedge J \wedge J-\frac{1}{\ell_{s}^{2}} \int_{X} J \wedge \frac{1}{4(2 \pi)^{2}}\left(\operatorname{tr} \bar{F}_{1,2}^{2}-\frac{1}{2} \operatorname{tr} \bar{R}^{2}\right) \\
& +\frac{1}{\ell_{s}^{2}} \sum_{a} N_{a}\left(\frac{1}{4} \mp \lambda_{a}\right) \int_{\Gamma_{a}} J .
\end{aligned}
$$

The first term is the tree-level gauge coupling and receives one-loop theshold corrections depending both on the Kähler moduli of the Calabi-Yau and the fivebrane moduli $\lambda_{a}$ (see also [43]). If we set all five-brane moduli to zero, then we 
nevertheless get a five-brane contribution of $1 / 4$ to the one-loop gauge couplings in both the first and the second $E_{8}$. From the Horava-Witten point of view this means that for $\lambda_{a}=0$, the five-brane is placed exactly in the middle between the two end of the world nine-branes and $\lambda_{a}$ is measured with respect to this symmetric configuration (see figure 2). We will give further evidence for this interpretation momentarily.

The next-to-leading order M-theory computation carried out in $[52,53]$ provides an $\mathcal{O}\left(\lambda^{2}\right)$ correction to the real part of the dilaton superfield

$$
S=\frac{1}{2 \pi}\left[e^{-2 \phi_{10}} \frac{\operatorname{Vol}(X)}{\ell_{s}^{6}}+\sum_{a} N_{a} \frac{\lambda_{a}^{2}}{2 \ell_{s}^{2}} \int_{\Gamma_{a}} J+i b_{0}^{(0)}\right] .
$$

This correction was derived in [53] essentially by requiring that the kinetic terms for the self-dual two-form on the M5-brane can indeed be correctly incorporated into an appropriate Kähler potential. Using this result and holomorphy of the gauge kinetic function leads to the gauge couplings

$$
\begin{aligned}
\frac{4 \pi}{g_{1,2}^{2}}= & \frac{1}{3 \ell_{s}^{6} g_{s}^{2}} \int_{X} J \wedge J \wedge J-\frac{1}{\ell_{s}^{2}} \int_{X} J \wedge\left(\operatorname{ch}_{2}\left(V_{N_{1,2}}\right)+\frac{1}{2} \sum_{n_{1,2}=1}^{M_{1,2}} c_{1}^{2}\left(L_{n_{1,2}}\right)+\right. \\
& \left.\frac{1}{2} c_{2}(T)\right)+\frac{1}{\ell_{s}^{2}} \sum_{a} N_{a}\left(\frac{1}{2} \mp \lambda_{a}\right)^{2} \int_{\Gamma_{a}} J .
\end{aligned}
$$

Note that the one-loop threshold corrections for the non-abelian gauge groups are universal inside each $E_{8}$ wall. For $\lambda_{a}=-\frac{1}{2}$, the contribution of the five-brane to the threshold corrections from $E_{8}^{(1)}$ is precisely that of a small instanton inside $E_{8}^{(1)}$. This unambiguously identifies $\lambda_{a}$ as the relative position of the five-brane measured with respect to the middle of the interval between the orbifold planes, as suggested already. We point out once more that different normalisations of the counter terms (18) would have resulted in a corresponding redefinition of $\lambda_{a}$. Moreover, as expected, if one places the five-brane inside the $E_{8}^{(2)}$ wall, its gauge threshold corrections to the gauge couplings from $E_{8}^{(1)}$ vanish and vice versa.

For the abelian gauge groups things are slightly different. The resulting in general non-diagonal abelian gauge couplings are given by

$$
\begin{aligned}
4 \pi \operatorname{Re}\left(f_{m_{i}, n_{i}}\right)= & \frac{\eta_{m_{i}, n_{i}}}{12 \ell_{s}^{6} g_{s}^{2}} \int_{X} J \wedge J \wedge J-\frac{\eta_{m_{i}, n_{i}}}{4 \ell_{s}^{2}} \int_{X} J \wedge\left(\operatorname{ch}_{2}\left(V_{N_{i}}\right)+\frac{1}{2} \sum_{k_{i}=1}^{M_{i}} c_{1}^{2}\left(L_{k_{i}}\right)+\right. \\
& \left.\frac{1}{2} c_{2}(T)\right)-\frac{1}{12 \ell_{s}^{2}} \int_{X} J \wedge\left(\sum_{p_{i}, q_{i}=1}^{M_{i}} \kappa_{m_{i}, p_{i}} \kappa_{n_{i}, q_{i}} c_{1}\left(L_{p_{i}}\right) c_{1}\left(L_{q_{i}}\right)\right) \\
& +\frac{\eta_{m_{i}, n_{i}}}{4 \ell_{s}^{2}} \sum_{a} N_{a}\left(\frac{1}{2} \mp \lambda_{a}\right)^{2} \int_{\Gamma_{a}} J
\end{aligned}
$$




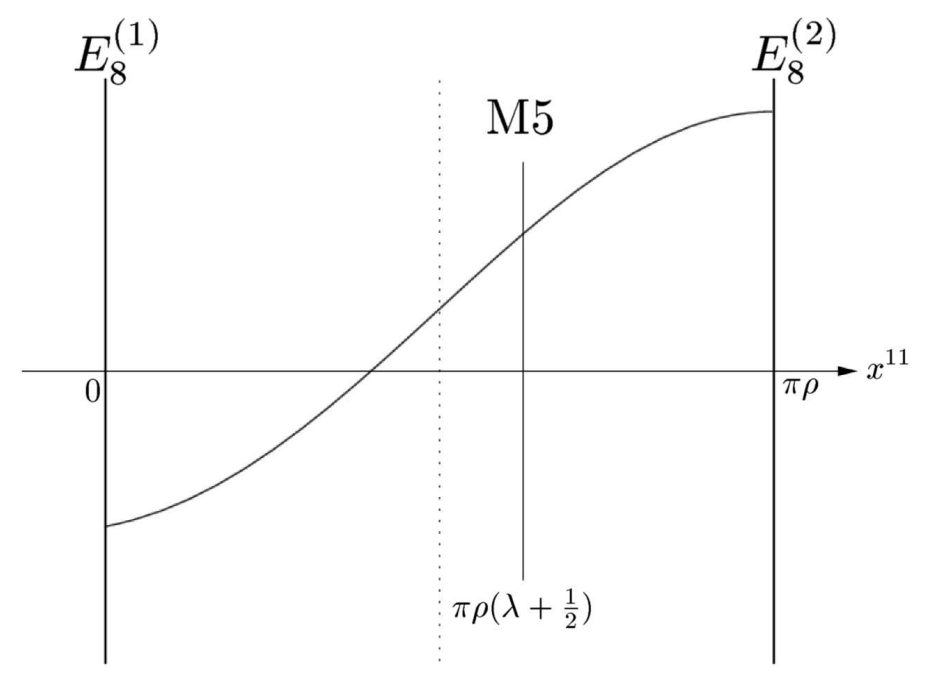

Figure 2: M5-brane potential in Horava-Witten theory on the Quintic induced by abelian gauge flux on $E_{8}^{(1)}$.

for both $U(1)$ factors from the same $E_{8}$ factor and by

$4 \pi \operatorname{Re}\left(f_{m_{1}, n_{2}}\right)=4 \pi f_{m_{1}, n_{2}}=\frac{1}{24 \ell_{s}^{2}} \int_{X} J \wedge\left(\sum_{p_{1}=1}^{M_{1}} \sum_{q_{2}=1}^{M_{2}} \kappa_{m_{1}, p_{1}} \kappa_{n_{2}, q_{2}} c_{1}\left(L_{p_{1}}\right) c_{1}\left(L_{q_{2}}\right)\right)$

for one $U(1)$ from the first and one $U(1)$ from the second $E_{8}$ (cf (133) for notation). Apparently, only for trivial line bundles, i.e. Wilson lines, do the extra threshold corrections vanish.

\subsection{Fayet-Iliopoulos terms}

Whenever we are dealing with anomalous $U(1)$ gauge factors, there are potential Fayet-Iliopoulos (FI) terms generated (e.g. [54]). With the help of the standard supersymmetric field theory formula

$$
D_{m} \frac{\xi_{m}}{g_{m}^{2}}=\left.D_{m} \frac{\partial \mathcal{K}}{\partial V_{m}}\right|_{V=0}
$$

the FI parameters $\xi_{m}$ can be computed from the Kähler potential $\mathcal{K}$. The Kähler potential in turn is of course determined by requiring that it reproduces the various kinetic terms in the four-dimensional action in the Einstein frame. The kinetic terms of the M5-brane action do not only induce the quadratic correction (43) in the definition of the superfield $S$, but also dictate a modification of the standard contribution $-\ln \left(S+S^{*}\right)$ to $\mathcal{K}$ by a term quadratic in $\Lambda_{a}+\Lambda_{a}^{*}[52,53]$. Furthermore, the various axionic couplings of the abelian gauge fields induce 
additional modifications of $\mathcal{K}$ such that these terms are recovered in the superfield formalism in the same way as the kinetic terms. In supergravity language, the rationale behind this is that the $\mathcal{N}=1$ supergravity gets gauged by the axionic shift symmetry; by a standard procedure the Kähler potential has to be rendered invariant under this gauging. As a result, the correct Kähler potential takes the form

$$
\begin{gathered}
\mathcal{K}=-\frac{M_{p l}^{2}}{8 \pi} \ln \left[S+S^{*}-\sum_{m} Q_{0}^{m} V_{m}+\sum_{a} \frac{N_{a}}{2} \frac{\left(\Lambda_{a}+\Lambda_{a}^{*}-\sum_{m} Q_{a}^{m} V_{m}\right)^{2}}{\left(\bar{\gamma}_{a}\right)_{k}\left(T_{k}+T_{k}^{*}-\sum_{a} Q_{k}^{m} V_{m}\right)}\right] \\
-\frac{M_{p l}^{2}}{8 \pi} \ln \left[-\sum_{i, j, k=1}^{h_{11}} \frac{d_{i j k}}{6}\left(T_{i}+T_{i}^{*}-\sum_{m} Q_{i}^{m} V_{m}\right)\left(T_{j}+T_{j}^{*}-\sum_{m} Q_{j}^{m} V_{m}\right)\right. \\
\left.\left(T_{k}+T_{k}^{*}-\sum_{m} Q_{k}^{m} V_{m}\right)\right] .
\end{gathered}
$$

Here $\frac{M_{p l}^{2}}{8 \pi}=\kappa_{10}^{-2} \operatorname{Vol}(X)$ and $V_{m}$ denotes the vector superfields. Note that for $V_{m}=0$ this is essentially the result derived in $[52,53]$. The charges $Q_{k}^{m}$ can be identified as the couplings in the mass terms (34),(35),(36) using the definition

$$
S_{\text {mass }}=\sum_{m=1}^{M} \sum_{k=0}^{h_{11}} \frac{Q_{k}^{m}}{2 \pi \alpha^{\prime}} \int_{\mathbb{R}_{1,3}} f_{m} \wedge b_{k}^{(2)}+\sum_{m=1}^{M} \sum_{a} \frac{Q_{a}^{m}}{2 \pi \alpha^{\prime}} \int_{\mathbb{R}_{1,3}} f_{m} \wedge \widetilde{b}_{a}^{(2)} .
$$

We finally obtain for the Fayet-Iliopoulos term

$$
\begin{gathered}
\frac{\xi_{m_{i}}}{g_{m_{i}}^{2}}=-\frac{1}{8 \ell_{s}^{6}} \sum_{n_{i}=1}^{M_{i}} \kappa_{m_{i}, n_{i}}\left[\int_{X} J \wedge J \wedge \frac{\bar{f}_{n_{i}}}{2 \pi}-e^{2 \phi_{10}} \ell_{s}^{4} \int_{X} \frac{\bar{f}_{n_{i}}}{2 \pi} \wedge \frac{1}{4(2 \pi)^{2}}\left(\operatorname{tr} \bar{F}_{i}^{2}-\frac{1}{2} \operatorname{tr} \bar{R}^{2}\right)\right. \\
\left.+e^{2 \phi_{10}} \ell_{s}^{4} \sum_{a} N_{a}\left(\frac{1}{2} \mp \lambda_{a}\right)^{2} \int_{\Gamma_{a}} \frac{\bar{f}_{n_{i}}}{2 \pi}\right] .
\end{gathered}
$$

Thus we learn that a flux through the two-cycle $\Gamma_{a}$ of a five-brane on the wall $E_{8}^{(i)}$ generates a one-loop D-term potential for the five-brane modulus $\lambda_{a}$. From (50) it seems at first sight that this D-term repels the five-brane from the wall and vanishes only if the five-brane lies on top of the other wall. We will come back to this issue in section 2.7 .

The FI-term (50) generalizes the one-loop corrected DUY equation in the presence of M5-branes to

$$
\begin{aligned}
& \int_{X} J \wedge J \wedge c_{1}\left(L_{m_{i}}\right)-\ell_{s}^{4} g_{s}^{2} \int_{X} c_{1}\left(L_{m_{i}}\right) \wedge\left(\operatorname{ch}_{2}\left(V_{N_{i}}\right)+\frac{1}{2} \sum_{n_{i}=1}^{M_{i}} c_{1}^{2}\left(L_{n_{i}}\right)+\right. \\
& \left.\frac{1}{2} c_{2}(T)\right)+\ell_{s}^{4} g_{s}^{2} \sum_{a} N_{a}\left(\frac{1}{2} \mp \lambda_{a}\right)^{2} \int_{\Gamma_{a}} c_{1}\left(L_{m_{i}}\right)=0 .
\end{aligned}
$$


In general, these conditions provide constraints fixing combinations of the Kähler moduli, the dilaton and five-brane moduli.

Let us pause a moment to comment on the physical interpretation of the fivebrane contribution to the above D-term. Arising at one loop in the weakly coupled heterotic string, it is expected to be due to appropriate amplitudes from membranes after unfolding the wrapped eleventh dimension in the strongly-coupled Horava-Witten regime. In fact, as derived in [53], there are non-perturbative contributions to the F-term superpotential from open membranes stretching between one of the orbifold fixed planes and the M5-brane provided that the worldvolume of the membranes is precisely of the form $I \times \Gamma_{a}$. Here $I$ simply denotes the interval along the eleventh dimension between the orbifold plane and fivebrane and $\Gamma_{a}$ is as always the internal cycle wrapped by the M5-brane. We see that, apparently, such configurations also contribute to the D-term potential if the membrane can couple to some abelian background gauge flux on the orbifold plane. As is manifest in (51), this can only happen if the five-brane wraps a two-cycle which, pulled back to the end of the world, carries non-vanishing gauge flux. In particular, this interpretation explains why the five-brane is sensitive to the presence of the gauge flux along $\Gamma_{a}$ even though it may be placed at an arbitrary position along the eleventh dimension: The presence of the gauge flux is communicated by the exchange of appropriate open membranes.

\subsection{D-term potential for M5-branes}

Let us discuss in a simple example what the effect of the FI terms is for the M5brane modulus $\lambda$. It is instructive to consider the Quintic Calabi-Yau manifold, which has only one Kähler modulus, and we assume that we have chosen a vector bundle $V \oplus L^{-1}$ embedded into the first $E_{8}$ wall without any matter charged under the $U(1)$. Then the D-term potential arising from the FI-term of the $U(1)$ is simply

$$
V_{D}=\frac{1}{2} g^{2}\left(\frac{\xi}{g^{2}}\right)^{2},
$$

where $g$ denotes the gauge coupling of the $U(1)$. For the Quintic one has $c_{2}(T)=$ $10 \eta^{2}$ and $J=\ell_{s}^{2} r \eta$ with $r>0$ in terms of the single $(1,1)$-form $\eta$. Moreover, we write $\operatorname{ch}_{2}(V)=-v \eta^{2}+\frac{1}{2} l^{2} \eta^{2}$ and $\operatorname{ch}_{2}(L)=\frac{1}{2} l^{2} \eta^{2}$ and introduce one five-brane wrapping the class $\gamma$. The tadpole cancellation condition then reads

$$
-v+l^{2}-\gamma^{2}=-10 .
$$

The relevant D-term potential takes the form

$$
V_{D} \simeq \frac{\left(\frac{r^{2}}{g_{s}^{2}}-\left(\gamma^{2}-5\right)+\left(\frac{1}{2}-\lambda\right)^{2} \gamma^{2}\right)^{2}}{\left(\frac{r^{2}}{g_{s}^{2}}-3\left(\gamma^{2}-5\right)+3\left(\frac{1}{2}-\lambda\right)^{2} \gamma^{2}-\frac{\kappa_{1,1}^{2}}{\eta_{1,1}} l^{2}\right)} .
$$


For fixed string coupling $g_{s}=0.5$, radius $r=2$ and parameters $\gamma=l=2$, $\kappa_{1,1}^{2} / \eta_{1,1}=1 / 10$, this potential for the five-brane modulus $\lambda$ has the characteristic shape shown in figure 2. Naively from the FI-term one might have expected that the five-brane is repelled by the $E_{8}$ walls carrying a non-trivial line bundle. However, the contribution of the $g^{2}$ term multiplying the FI-term in the scalar potential changes this picture and leads to an attractive potential between the five-brane and the $E_{8}$ wall carrying the bundle. This is actually well in agreement with the interpretation of the D-term potential as being due to open membranes stretching between the orbifold fixed plane and the M5-brane: Their contribution is of course minimized precisely if the interval along which they wrap between the end of the world and the five-brane is vanishing.

\section{$3 \quad$ Flipped $S U(5) \times U(1)_{X}$}

We are finally in a position to discuss the central part of this article, the application of the formalism introduced so far to the construction of GUT like heterotic string compactifications. As pointed out in the introduction, what has been discussed in detail so far in the literature is the following standard realisation of GUT gauge groups. On the Calabi-Yau one chooses an $S U(4)$ or $S U(5)$ bundle embedded into one of the two $E_{8}$ factors. The resulting observable gauge groups are $S O(10)$ or $S U(5)$, respectively. In addition one gets chiral matter transforming in the $(\mathbf{1 6})$ or $(\mathbf{1 0})+(\overline{\mathbf{5}})$ representation of the gauge group. However, in these scenarios there does not appear a Higgs field that can break the GUT group down to the Standard Model. This is achieved by breaking $S O(10)$ or $S U(5)$ via nontrivial discrete Wilson lines, which in general can only exist if the fundamental group of the Calabi-Yau is non-trivial. Such Calabi-Yau's can be constructed by taking free discrete quotients of a Calabi-Yau with vanishing fundamental group. The electroweak Higgs can appear from the $(\mathbf{1 0})$ or the $(\mathbf{5})+(\overline{\mathbf{5}})$ representations. Without doubt, from the physical point of view, this is a very simple and compelling picture and recently models whose particle spectrum is quite close to the Standard Model have been constructed [3,8,10].

As mentioned above, in this construction the breaking of the gauge symmetry down to the Standard Model is achieved via discrete Wilson lines, in more mathematical terms by flat abelian bundles. In this section we would like to investigate whether one can use also non-flat line bundles to obtain phenomeno-

logically interesting GUT models. We will see that we will almost directly be led to considering flipped $S U(5)$ models.

\section{1 $S U(4) \times U(1)$ bundles}

Instead of first using an $S U(4)$ bundle and then breaking $S O(10)$ down to flipped $S U(5)$ by a discrete Wilson line, we consider a bundle 
with structure group $S U(4) \times U(1)$ on a Calabi-Yau $X$ with $\pi_{1}(X)=0$. Such types of construction have been considered in [16] before and further details of this particular one can be found in [23] (see also the recent articles $[35,36]$ ).

The starting point is a bundle

$$
W=V \oplus L^{-1}, \quad \text { with } c_{1}(V)=c_{1}(L), \operatorname{rank}(V)=4,
$$

with structure group $S U(4) \times U(1)$. This bundle $W$ can now be embedded into an $S U(5)$ subgroup of $E_{8}$ so that the commutant is $S U(5) \times U(1)_{1}$. We embed the $U(1)$ bundle such that the charge matrix is

$$
\mathcal{Q}_{1}=(1,1,1,1,-4)
$$

in the notation of [23]. In fact, consider the breaking of the original structure group $S U(5) \rightarrow U(4) \times U(1)$ and the corresponding decomposition of $(\mathbf{5}, \mathbf{1 0}) \rightarrow$ $(\mathbf{4}, \mathbf{1 0})_{-1}+(\mathbf{1}, \mathbf{1 0})_{4}$ to read off the unique charge assignments of $V$ and $L$. Using the decomposition of the adjoint representation of $E_{8}$

$$
\mathbf{2 4 8} \stackrel{(\mathbf{1 5}, \mathbf{1})_{0}}{ } \underset{ }{\longrightarrow}\left\{\begin{array}{c}
(\mathbf{1}, \mathbf{1})_{0}+(\mathbf{1}, \mathbf{1 0})_{4}+(\mathbf{1}, \overline{\mathbf{1 0}})_{-4}+(\mathbf{1}, \mathbf{2 4})_{0} \\
(\mathbf{4}, \mathbf{1})_{-5}+(\mathbf{4}, \overline{\mathbf{5}})_{3}+(\mathbf{4}, \mathbf{1 0})_{-1} \\
(\overline{\mathbf{4}}, \mathbf{1})_{5}+(\overline{\mathbf{4}}, \mathbf{5})_{-3}+(\overline{\mathbf{4}}, \overline{\mathbf{1 0}})_{1} \\
(\mathbf{6}, \mathbf{5})_{2}+(\mathbf{6}, \overline{\mathbf{5}})_{-2}
\end{array}\right\}
$$

we list the resulting massless spectrum in Table 1.

\begin{tabular}{|c||c|c|}
\hline \hline$S U(5) \times U(1)_{1}$ & bundle & SM part. \\
\hline \hline $\mathbf{1 0}_{-1}$ & $V$ & $\left(q_{L}, d_{R}^{c}, \nu_{R}^{c}\right)+\left[H_{10}+\bar{H}_{10}\right]$ \\
$\mathbf{1 0}_{4}$ & $L^{-1}$ & - \\
\hline$\overline{\mathbf{5}}_{3}$ & $V \otimes L^{-1}$ & $\left(u_{R}^{c}, l_{L}\right)$ \\
$\overline{\mathbf{5}}_{-2}$ & $\wedge^{2} V$ & {$\left[\left(h_{3}, h_{2}\right)+\left(\bar{h}_{3}, \bar{h}_{2}\right)\right]$} \\
\hline $\mathbf{1}_{-5}$ & $V \otimes L$ & $e_{R}^{c}$ \\
\hline
\end{tabular}

Table 1: Massless spectrum of $H=S U(5) \times U(1)_{1}$ models.

The massless fields precisely carry, up tp a common factor, the $U(1)_{X}$ charges as appearing in the flipped $S U(5)[24,25], Q_{X}=-\frac{1}{2} Q_{1} \cdot{ }^{9}$ Recall that this model

\footnotetext{
${ }^{9}$ Note that the normalisation of $Q_{X}$, as chosen here, differs from the one in [24] by a factor of $\frac{1}{2}$.
} 
differs from the conventional Georgi-Glashow GUT scenario in that the $U(1)_{Y}$ is not entirely contained in the $S U(5)$, but arises as the specific linear combination

$$
\frac{1}{2} Q_{Y}=-\frac{1}{5} Q_{Z}+\frac{2}{5} Q_{X}
$$

where $Z$ is the generator of $S U(5)$ commmuting with the generators of the Standard Model $S U(3) \times S U(2)$. In the normalisation of [24] $Z$ is given by $Z=\operatorname{diag}(-1 / 3,-1 / 3,-1 / 3,1 / 2,1 / 2)$. The way how the MSSM matter organizes into flipped $S U(5)$ multiplets is related to the Georgi-Glashow scenario by the flip

$$
d_{R}^{c} \leftrightarrow u_{R}^{c}, \quad e_{R}^{c} \leftrightarrow \nu_{R}^{c} .
$$

Most importantly, the $(\mathbf{1 0})_{-1}$ contains the right-handed neutrino as a particle uncharged under the MSSM $S U(3) \times S U(2) \times U(1)_{Y}$, and giving it a VEV can therefore serve as the Higgs effect breaking the GUT group down to the Standard Model one. It is this peculiarity of flipped $S U(5)$ which at first sight allows us to work on manifolds without Wilson lines. However, if we only consider the bundle (55) inside the first $E_{8}$ with $c_{1}(L) \neq 0$, one Kähler/dilaton modulus receives a mass from the DUY constraint and therefore also one axion in combination with the $U(1)_{X}$ gauge boson. Therefore, after GUT Higgsing by $H_{10}$ the resulting $U(1)_{Y}$ would also be massive. This seems to bring us back into the old situation that we are forced to consider manifolds with non-vanishing fundamental group to allow for non-trivial flat bundles ${ }^{10}$.

Alternatively, here we propose to embed a second line bundle into the other $E_{8}$, such that a linear combination of the two observable $U(1)$ 's remains massless. Concretely, we embed the line bundle $L$ (or more precisely $L \oplus L^{-1}$ ) also in the second $E_{8}$, where it leads to the breaking $E_{8} \rightarrow E_{7} \times U(1)_{2}$ and the decomposition

$$
\mathbf{2 4 8} \stackrel{E_{7} \times U(1)}{\longrightarrow}\left\{(\mathbf{1 3 3})_{0}+(\mathbf{1})_{0}+(\mathbf{5 6})_{1}+(\mathbf{5 6})_{-1}+(\mathbf{1})_{2}+(\mathbf{1})_{-2}\right\} .
$$

The resulting massless spectrum is displayed in Table 2. The tadpole cancellation

\begin{tabular}{|c||c|}
\hline \hline$E_{7} \times U(1)_{2}$ & bundle \\
\hline \hline $\mathbf{5 6}_{1}$ & $L^{-1}$ \\
$\mathbf{1}_{2}$ & $L^{-2}$ \\
\hline
\end{tabular}

Table 2: Massless spectrum of $H=E_{7} \times U(1)_{2}$ models.

\footnotetext{
${ }^{10}$ For $\pi_{1}(X)=0$, a line bundle with $c_{1}(L)=0$ is always trivial and the observable gauge group gets enhanced to $S O(10)$.
} 
condition for this model reads

$$
\operatorname{ch}_{2}(V)+3 \operatorname{ch}_{2}(L)-\sum_{a} N_{a} \bar{\gamma}_{a}=-c_{2}(T) .
$$

Considering the mass terms in (34) and (35) and computing

$$
\kappa_{1,1}=-10, \quad \kappa_{2,2}=-4,
$$

one realizes that the linear combination

$$
U(1)_{X}=-\frac{1}{2}\left(U(1)_{1}-\frac{5}{2} U(1)_{2}\right)
$$

remains massless if the following conditions are satisfied

$$
\int_{X} c_{1}(L) \wedge c_{2}(V)=0, \quad \int_{\Gamma_{a}} c_{1}(L)=0 \quad \text { for all M5 branes. }
$$

The resulting chiral massless spectrum simplifies considerably and is given in Table 3 .

\begin{tabular}{|c||c|c|}
\hline \hline$S U(5) \times U(1)_{X} \times E_{7}$ & bundle & SM part. \\
\hline \hline$(\mathbf{1 0}, \mathbf{1})_{\frac{1}{2}}$ & $\chi(V)=g$ & $\left(q_{L}, d_{R}^{c}, \nu_{R}^{c}\right)+\left[H_{10}+\bar{H}_{10}\right]$ \\
$(\mathbf{1 0}, \mathbf{1})_{-2}$ & $\chi\left(L^{-1}\right)=0$ & - \\
\hline$(\overline{\mathbf{5}}, \mathbf{1})_{-\frac{3}{2}}$ & $\chi\left(V \otimes L^{-1}\right)=g$ & $\left(u_{R}^{c}, l_{L}\right)$ \\
$(\overline{\mathbf{5}}, \mathbf{1})_{1}$ & $\chi\left(\bigwedge^{2} V\right)=0$ & {$\left[\left(h_{3}, h_{2}\right)+\left(\bar{h}_{3}, \bar{h}_{2}\right)\right]$} \\
\hline$(\mathbf{1}, \mathbf{1})_{\frac{5}{2}}$ & $\chi(V \otimes L)+\chi\left(L^{-2}\right)=g$ & $e_{R}^{c}$ \\
\hline$(\mathbf{1}, \mathbf{5 6})_{\frac{5}{4}}$ & $\chi\left(L^{-1}\right)=0$ & - \\
\hline
\end{tabular}

Table 3: Massless spectrum of $H=S U(5) \times U(1)_{X}$ models with $g=\frac{1}{2} \int_{X} c_{3}(V)$.

Therefore, one gets precisely $g$ generations of flipped $S U(5)$ matter. Note that in general the right-handed electrons receive contributions from both the first and the second $E_{8}$. From a phenomenological point of view, we want to circumvent these latter in order to avoid non-MSSM like selection rules for their Yukawa couplings. They are absent if additionally one requires

$$
\int_{X} c_{1}^{3}(L)=0
$$


If the tadpole condition is satisfied, this implies, together with (64), that also $\int_{X} c_{1}(L) \wedge c_{2}(T)=0$. With these extra conditions, the generalized DUY condition for the bundle $L$ simplifies considerably,

$$
\int_{X} J \wedge J \wedge c_{1}(L)=0
$$

and contains only the tree-level part. Therefore, it freezes only one of the $h_{11}$ Kähler moduli. By contrast, the threshold corrections to the gauge kinetic functions will be non-vanishing. For consistency of the low-energy effective theory we need to ensure that the DUY can actually be solved in a regime inside the Kähler cone where the real part of the threshold corrected gauge kinetic functions is positive, at least for the unbroken gauge symmetries. Apart form the $S U(5)$ and the hidden $E_{7}$ symmetry, we will therefore have to check this condition for the gauge kinetic function of the generator of $U(1)_{X}$, which is given by

$$
f_{X, X}=\frac{1}{4}\left(f_{1,1}+\left(\frac{5}{2}\right)^{2} f_{2,2}+5 f_{1,2}\right)
$$

in terms of the the corresponding objects for $U(1)_{1}$ and $U(1)_{2}$.

\subsection{Yukawa couplings and proton decay}

This string theory realization of flipped $S U(5) \times U(1)_{X}$ features many, though not all, of the characteristic features of the field theory GUT model. For their details we refer to $[24,25,33]$. In particular, the GUT breaking via a non-vanishing vacuum expectation value of the singlet component in $H_{10}+\bar{H}_{10}$ leads to a natural solution of the doublet-triplet splitting problem via a missing partner mechanism in the superpotential coupling

$$
10_{\frac{1}{2}}^{H} 10_{\frac{1}{2}}^{H} 5_{-1} .
$$

Therefore, problematic dimension-five operators mediating proton decay can be suppressed. Moreover, the gauge invariant Yukawa couplings

$$
10_{\frac{1}{2}}^{i} 10_{\frac{1}{2}}^{j} \mathbf{5}_{-1}, \quad \mathbf{1 0} \frac{0_{\frac{1}{2}}^{i}}{\overline{5}_{-\frac{3}{2}}^{j}} \overline{\mathbf{5}}_{1}, \quad \overline{\mathbf{5}}_{-\frac{3}{2}}^{i} \mathbf{1}_{\frac{5}{2}}^{j} \mathbf{5}_{-1},
$$

lead to Dirac mass-terms for the $d,(u, \nu)$ and $e$ quarks and leptons after electroweak symmetry breaking. If there exist additional gauge singlets $\Phi$, then

couplings of the form $\mathbf{1 0} \frac{1}{\frac{1}{2}} \overline{\mathbf{1 0}}_{-\frac{1}{2}}^{H} \Phi$ can give rise to Majorana type neutrino masses and therefore to a see-saw mechanism.

Since the electroweak Higgs carries different quantum numbers than the lepton doublet, the dangerous dimension-four proton decay operators

$$
\text { lle } \in \overline{5}_{-\frac{3}{2}}^{i} \mathbf{1}_{\frac{5}{2}}^{j} \overline{5}_{-\frac{3}{2}}^{k}, \quad \text { qdl, udd } \in \mathbf{1 0}_{\frac{1}{2}}^{i} \mathbf{1 0}_{\frac{1}{2}}^{j} \overline{5}_{-\frac{3}{2}}^{k}
$$


are not gauge invariant and thus absent. A detailed discussion of this peculiar property of heterotic constructions with line bundles has recently been given in [35] in the context of Georgi-Glashow $S U(5)$. Furthermore, as shown in [55], flipped $S U(5)$ differs from the Georgi-Glashow model in that also the dimensionsix proton decay operators, emerging after integrating out the off-diagonal gauge bosons in the $(\mathbf{3}, \mathbf{2})$, can be completely eliminated. Additional details and more references can also be found in [56].

The issue of gauge coupling unification for this model is absolutely identical to the discussion to be presented in section 5.2. Due to the embedding of $U(1)_{X}$ into both $E_{8}$ walls, the tree level gauge couplings satisfy the relation $\alpha_{3}=\alpha_{2}=\frac{8}{3} \alpha_{Y}$ instead of the usual factor $5 / 3$. Therefore, with the minimal supersymmetric matter content the tree level gauge couplings do not unify at $M_{G U T}=2 \cdot 10^{16} \mathrm{GeV}$. What rescues us are the one-loop threshold corrections to be further discussed in section 5.2 .

\section{Three-generation models}

It is now time to construct explicit examples of our framework with three chiral generations. This will be done choosing as the background manifold an elliptically fibered Calabi-Yau threefold, where we can exploit the spectral cover construction $[28,29]$ to endow it with stable holomorphic vector bundles. Let us first recapitulate briefly the most important aspects of such a construction (see also the account in [57] for further details). The reader familiar with this technique may wish to jump directly to section 4.3 .

\subsection{Elliptically fibered Calabi-Yau manifolds}

An elliptically fibered Calabi-Yau three-fold $X$ consists of a complex two-surface $B$ as base space, together with an analytic map

$$
\pi: X \rightarrow B
$$

where fibers over each point $b$ in the base

$$
E_{b}=\pi^{-1}(b)
$$

are elliptic curves, which can be described by the homogeneous Weierstrass equation

$$
z y^{2}=4 x^{3}-g_{2} x z^{2}-g_{3} z^{3}
$$

Additionally, we require $X$ to admit a global section $\sigma: B \rightarrow X$, assigning to every point in the base $b \in B$ the zero element $\sigma(b)=p \in E_{b}$ on the fiber. We denote by $\mathcal{L}$ the conormal bundle to the section $\sigma(B)$. One can regard $g_{2}$ and $g_{3}$ 
as sections of $\mathcal{L}^{4}$ and $\mathcal{L}^{6}$, respectively, where $\mathcal{L}^{i}$ is the $i$-fold tensor product of $\mathcal{L}$ with itself.

The Calabi-Yau condition requires, besides the fact that the base space and the fibers have to be complex manifolds themselves, that the first Chern class of the tangent bundle $T$ must vanish,

$$
c_{1}(T)=0 \text {. }
$$

This implies $\mathcal{L}=K_{B}^{-1}$, where $K_{B}$ is the canonical bundle of the base space.

It follows that $K_{B}^{-4}$ and $K_{B}^{-6}$ must have sections $g_{2}$ and $g_{3}$, respectively. The surfaces compatible with this condition are found to be del Pezzo, Hirzebruch, Enriques und blow-ups of Hirzebruch surfaces.

It has been shown that on such spaces the Chern classes of the tangent bundle of the total space are given by the Chern classes of the base space. Especially, we need later the second Chern class of the tangent bundle:

$$
c_{2}(T)=12 \sigma c_{1}(B)+11 c_{1}(B)^{2}+c_{2}(B),
$$

where $\sigma$ is the Poincaré dual two-form to the global section.

\subsection{Spectral cover construction}

A $U(n)$ or $S U(n)$ vector bundle over an elliptically fibered Calabi-Yau space $X$ can be obtained via the so-called spectral cover construction. The starting point is the observation that a vector bundle on an elliptic curve splits into a direct sum of line bundles

$$
\mathcal{V}=\mathcal{N}_{1} \oplus \ldots \oplus \mathcal{N}_{n}
$$

Since finally, the vector bundle should be supersymmetric, it must admit a field strength satifying the hermitian Yang-Mills equations, which is equivalent to the requirement that the associated bundle is holomorphic $\mu$-semi-stable. Therefore, the line bundles $\mathcal{N}_{i}$ must be of degree zero. On an elliptic curve this means that there is a unique point $Q_{i}$ such that there is a meromorphic section of $\mathcal{N}_{i}$ with a pole at $Q_{i}$ and with a zero at the origin $p$. Thus, the vector bundle on the curve is determined by an unordered $n$-tuple of points on the curve.

In intuitive terms, on an elliptically fibered space a vector bundle is then determined by a set of $n$ points, varying over the base, i.e. an $n$-fold cover of $B$ with $\pi_{C}: C \rightarrow B$. This is called the spectral cover, determining the vector bundle on the fibers. One has additionally to specify one more line bundle $\mathcal{N}$ such that $\pi_{C *} \mathcal{N}$ is a vector bundle on $B$ with the direct sum of $n$ lines $\left.\mathcal{N}\right|_{Q_{i}}$ as fibers. The whole vector bundle on $X$ is given using the Poincaré bundle $\mathcal{P}$, which is a line bundle over the fiber product $X \times{ }_{B} C$,

$$
\mathcal{V}=\pi_{1 \star}\left(\pi_{2}^{*} \mathcal{N} \otimes \mathcal{P}\right)
$$


Here $\pi_{1}$ and $\pi_{2}$ are the projections on the first and second factor of the fiber product, respectively.

Since for $U(n)$ bundles constructed in this way, the semi-stability is an intricate issue, we consider from now on only $S U(n)$ bundles and twist them finally with $U(1)$ bundles to obtain $U(n)$ bundles, which are $\mu$-semi-stable again . For an $S U(n)$-bundle we have the additional condition that the first Chern class must vanish. Friedman, Morgan and Witten [29] have found a formula for $c_{1}(\mathcal{V})$,

$$
c_{1}(\mathcal{V})=\pi_{C *}\left(c_{1}(\mathcal{N})+\frac{1}{2} c_{1}(C)-\frac{1}{2} \pi^{*} c_{1}(\mathcal{V})\right),
$$

where $c_{1}(C)$ and $c_{1}(B)$ are the first Chern classes of the tangent bundles of $C$ and $B$, respectively. Since $c_{1}(\mathcal{V})$ must vanish, we have

$$
c_{1}(\mathcal{N})=-\frac{1}{2} c_{1}(C)+\frac{1}{2} \pi_{C}^{*} c_{1}(B)+\gamma
$$

in terms of the cohomology class $\gamma$ satisfying

$$
\pi_{C *} \gamma=0
$$

In general, $\gamma$ can be writen as

$$
\gamma=\lambda\left(n \sigma-\pi_{C}^{*} \eta+n \pi_{C}^{*} c_{1}(B)\right)
$$

where $\lambda \in \mathbb{Q}$. Putting everything together, we have

$$
c_{1}(\mathcal{N})=n\left(\frac{1}{2}+\lambda\right) \sigma+\left(\frac{1}{2}-\lambda\right) \pi_{C}^{*} \eta+\left(\frac{1}{2}+n \lambda\right) \pi_{C}^{*} c_{1}(B) .
$$

Since $c_{1}(\mathcal{N})$ must be an integer class, not every value of $\lambda$ is allowed. One often uses the conditions:

$$
\begin{aligned}
n \quad \text { odd }: & \lambda \in \mathbb{Z}+\frac{1}{2}, & & \\
n \quad \text { even }: & \lambda \in \mathbb{Z}, & \eta+c_{1}(B)=0 & \bmod 2, \\
& \lambda \in \mathbb{Z}+\frac{1}{2}, & c_{1}(B)=0 & \bmod 2 .
\end{aligned}
$$

Note that these are only sufficient conditions. In our example, we also consider the more general case that $\lambda$ can be of higher fraction.

The $S U(n)$ bundles constructed so far are only $\mu$-semi-stable. It has been shown [28] that the spectral cover must be irreducible in order to obtain a $\mu$ stable one ${ }^{11}$, which imposes two more conditions to the curve $\eta$ [58]:

\footnotetext{
${ }^{11}$ In fact, the proof of stability assumes that the Kähler parameter of the fiber lies in a certain range near the boundary of the Kähler cone, i.e $J=\epsilon \sigma+J_{B}$ with sufficiently small $\epsilon$. Since the value of $\epsilon$ is not known, in all models involving the spectral cover constructions it is therefore a subtle issue if the region of stability overlaps with the perturbative regime, which is needed to have control over non-perturbative effects. In all examples which will be relevant for us, the constraints will leave us enough freedom to go to regions of the Kähler cone where $\epsilon$ is much smaller than $J_{B}$.
} 
- The linear system $|\eta|$ has to be base point free.

- The class $\eta-n c_{1}(B)$ has to be effective.

A $\mu$-stable $U(n)$ bundle can be obtained by twisting an $S U(n)$ bundle with an additional line bundle $\mathcal{Q}$ on $X$ with

$$
c_{1}(\mathcal{Q})=q \sigma+c_{1}(\zeta),
$$

where $c_{1}(\zeta) \in H^{2}(B, \mathbb{Z})$. The resulting $U(n)$ bundle

$$
V=\mathcal{V} \otimes \mathcal{Q}
$$

is $\mu$-stable if the original $S U(n)$ bundle is. Using $\operatorname{ch}(\mathcal{V} \otimes \mathcal{Q})=\operatorname{ch}(\mathcal{V}) \operatorname{ch}(\mathcal{Q})$, we can compute the resulting Chern characters of $V$ from the ones of $\mathcal{V}[59,60]$ and $\mathcal{Q}:$

$$
\begin{aligned}
\operatorname{ch}_{1}(V)= & n q \sigma+n c_{1}(\zeta) \\
\operatorname{ch}_{2}(V)= & {\left[-\eta+\frac{n q}{2}\left(2 c_{1}(\zeta)-q c_{1}(B)\right)\right] \sigma+a_{F}, } \\
\operatorname{ch}_{3}(V)= & \lambda \eta\left(\eta-n c_{1}(B)\right)-\eta c_{1}(\zeta)+q\left(\frac{n}{2} c_{1}(\zeta)^{2}-\omega\right)+ \\
& q c_{1}(B)\left(\eta-\frac{n q}{2} c_{1}(\zeta)+\frac{n q^{2}}{6} c_{1}(B)\right) .
\end{aligned}
$$

Here

$$
\begin{aligned}
a_{F} & =\frac{n}{2} c_{1}(\zeta)^{2}-\omega, \\
\omega & =-\frac{1}{24} c_{1}(B)^{2}\left(n^{3}-n\right)+\frac{1}{2}\left(\lambda^{2}-\frac{1}{4}\right) n \eta\left(\eta-n c_{1}(B)\right),
\end{aligned}
$$

where $\operatorname{ch}_{3}(V)$ has already been integrated over the fiber. The Chern classes read

$$
\begin{aligned}
c_{1}(V)= & n q \sigma+n c_{1}(\zeta), \\
c_{2}(V)= & {\left[\eta+n q(n-1)\left(c_{1}(\zeta)-\frac{q}{2} c_{1}(B)\right)\right] \sigma+\frac{n^{2}}{2} c_{1}(\zeta)^{2}-a_{F}, } \\
c_{3}(V)= & \frac{n q^{2}}{6}\left(n^{2}-3 n+2\right)\left(q c_{1}(B)^{2}-3 c_{1}(\zeta) c_{1}(B)\right), \\
& +\frac{n q}{2}\left(n^{2}-2 n+2\right) c_{1}(\zeta)^{2}+(2 q-n q-2 n \lambda) \eta c_{1}(B) \\
& +(n-2) \eta c_{1}(\zeta)+2 \lambda \eta^{2}-n q a_{F}-2 q \omega .
\end{aligned}
$$

To summarize, a $U(n)$ bundle is completely specified by the rational number $\lambda$, the integer $q$ and the classes $\eta$ and $c_{1}(\zeta)$. 


\subsection{An example on $\mathrm{dP}_{4}$}

The del Pezzo surfaces $\mathrm{dP}_{r}, r=0,1, \ldots, 8$ are obtained from the complex projective space $\mathbb{P}^{2}$ by blowing up $r$ points. Hence $H^{2}\left(\mathrm{dP}_{r}\right)$ is generated by $r+1$ elements: $l$, which is the class inherited from the projective space, and $E_{1}, \ldots, E_{r}$, generated by the blown-up points. The intersection numbers are

$$
\begin{aligned}
l \cdot l & =\int_{\mathrm{dP}_{r}} l \wedge l=1, \\
E_{i} \cdot E_{j} & =\int_{\mathrm{dP}_{r}} E_{i} \wedge E_{j}=-\delta_{i j}, \\
E_{i} \cdot l & =\int_{\mathrm{dP}_{r}} E_{i} \wedge l=0,
\end{aligned}
$$

and the Chern classes read

$$
c_{1}\left(\mathrm{dP}_{r}\right)=3 l-\sum_{m=1}^{r} E_{m}, \quad c_{2}\left(\mathrm{dP}_{r}\right)=3+r .
$$

The effective classes on del Pezzo surfaces form a cone, called Mori cone. The Mori cone is linearly generated by a finite set of effective classes if $r \leq 8$. The linear system of a curve $|\eta|$ is base point free if $\eta \cdot E \geq 0$ for every curve $E$ with $E^{2}=-1$ and $E \cdot c_{1}(B)=1$, which are precisely the generators of the Mori cone.

We turn now concretely to $\mathrm{dP}_{4}$ as the base space for an elliptically fibered Calabi-Yau space $X$. The second Chern class of the tangent bundle is given by (75),

$$
c_{2}(T)=\left[36 l-12 \sum_{i=1}^{4} E_{i}\right] \sigma+62 F,
$$

where $c_{1}\left(\mathrm{dP}_{4}\right)$ is expanded in the cohomological basis and $F$ is the class of the fiber. The Mori cone is generated by the 10 effective classes $E_{i}, l-E_{i}-E_{j}$, $i, j=1, \ldots, 4, i \neq j$.

We have finally introduced all the relevant technology for constructing interesting solutions. We have found a couple of three-generation flipped $S U(5)$ vacua satisfying all the required constraints. They are listed in Table 5 of Appendix B. We choose the following example to demonstrate their properties. The $U(4)$ bundle is given by the data

$$
\begin{aligned}
\lambda & =\frac{1}{4}, \quad q=0, \\
\eta & =14 l-2 E_{1}-6 E_{2}-6 E_{3}-2 E_{4}, \\
c_{1}(\zeta) & =-l+E_{2}+E_{3}+E_{4} .
\end{aligned}
$$


Note that the first Chern class of the line bundle $\mathcal{N}$ in the spectral cover constructon (82) is an integer class, as required:

$$
c_{1}(\mathcal{N})=3 \sigma+\pi_{C}^{*}\left(8 l-2 E_{1}-3 E_{2}-3 E_{3}-2 E_{4}\right) .
$$

It is easy to see that $|\eta|$ is base point free, since its intersection with the generators of the Mori cone is always positive. One can also easily show that $\eta$ is effective as well as $\eta-4 c_{1}\left(\mathrm{dP}_{4}\right)=2 l+2 E_{1}-2 E_{2}-2 E_{3}+2 E_{4}$. Thus, this bundle is $\mu$-stable. The resulting Chern classes are

$$
\begin{aligned}
& c_{1}(V)=-4 l+4 E_{2}+4 E_{3}+4 E_{4}, \\
& c_{2}(V)=\left[14 l-2 E_{1}-6 E_{2}-6 E_{3}-2 E_{4}\right] \sigma-29 F .
\end{aligned}
$$

In our setup, the first Chern class of the line bundle must be equal to the first Chern class of the vector bundle (see (55)), thus

$$
c_{1}(L)=-4 l+4 E_{2}+4 E_{3}+4 E_{4} .
$$

In our example model, we also include M5-branes. Their combined associated cohomology class is

$$
[W]=27 F+\left(22 l-10 E_{1}-6 E_{2}-6 E_{3}-10 E_{4}\right) \sigma
$$

To make physical sense, $[W]$ must be Poincaré dual to the homology class of a curve $\Gamma$ in $X$, and must be therefore effective. $[W]$ is effective if its part on the fiber is greater than or equal to zero and its part on the base is effective in $B$. Therefore, we rewrite $[W]$ in terms of generators of the Mori cone,

$$
\begin{aligned}
{[W]=} & \sum_{a} N_{a} \bar{\gamma}_{a}=27 F+\left[12 E_{1}+6\left(l-E_{1}-E_{2}\right)\right. \\
& \left.+6\left(l-E_{1}-E_{3}\right)+10\left(l-E_{1}-E_{4}\right)\right] \sigma
\end{aligned}
$$

The generators of the Mori cone, being irreducible as effective classes, represent the classes dual to the irreducible curves $\gamma_{a}$ around which we wrap $N_{a}$ five-branes. In general, this decomposition is in general not unique. However, we also have to satisfy the constraint $\int_{\Gamma_{a}} c_{1}(L)=0$ for a massless $U(1)_{X}$, and (103) is the only remaining decomposition compatible with this requirement. The tadpole cancellation condition (6) for this setup, written in terms of Chern classes, takes the form

$$
-c_{2}(V)+2 c_{1}^{2}(L)-[W]=-c_{2}(T)
$$

and is indeed satisfied. It is a simple calculation to show that the conditions to keep the $U(1)_{X}$ in the flipped $S U(5)$ model massless hold

$$
\int_{X} c_{1}(L) \wedge c_{2}(V)=0, \quad \int_{\Gamma_{a}} c_{1}(L)=\int_{X} c_{1}(L) \wedge \bar{\gamma}_{a}=0 .
$$


Since the Chern class of the line bundle has no part in the fiber, the integral over its third power trivially vanishes,

$$
\int_{X} c_{1}^{3}(L)=0
$$

and thus a contribution to the right-handed electrons from the second $E_{8}$ factor is prevented. The number of generations in our example is given by

$$
\chi(V)=\frac{1}{2} \int_{X} c_{3}(V)=3
$$

since $\int_{X} c_{1}(V) \wedge c_{2}(V)=\int_{X} c_{1}(L) \wedge c_{2}(V)=0$.

Expanding the Kähler class in the cohomological basis,

$$
J=l_{s}^{2}\left(r_{\sigma} \sigma+r_{0} l+\sum_{m=1}^{4} r_{m} E_{i}\right),
$$

the DUY-equation (66)

$$
\int_{X} J \wedge J \wedge c_{1}(L)=-8 l_{s}^{4} r_{\sigma}\left(r_{0}+r_{2}+r_{3}+r_{4}\right)=0
$$

fixes one Kähler modulus. There exist solutions inside the Kähler cone. Take as an example

$$
0<r_{\sigma}<2 \rho, \quad r_{0}=3 \rho, \quad r_{m}=-\rho, \quad m=1, \ldots, 4 .
$$

With this choice, equation (109) holds and the Kähler class lies inside the Kähler cone for every $\rho \in \mathbb{R}^{+}$.

The universal gauge coupling for the non-abelian visible gauge group (44) can be computed as ${ }^{12}$

$$
\frac{4 \pi}{g_{1}^{2}}=\frac{1}{3 g_{s}^{2}}\left(5 r_{\sigma}^{3}-15 r_{\sigma}^{2} \rho+15 r_{\sigma} \rho^{2}\right)-24 r_{\sigma}-4 \rho-\left(\frac{1}{2}-\lambda_{5}\right)^{2}\left(7 r_{\sigma}-34 \rho\right),
$$

which is positive for a suitable choice of parameters. The abelian gauge couplings are given by (4546)

$$
\begin{aligned}
4 \pi \operatorname{Re}\left(f_{i, i}\right)= & \frac{\eta_{i, i}}{4}\left(\frac{1}{3 g_{s}^{2}}\left(5 r_{\sigma}^{3}-15 r_{\sigma}^{2} \rho+15 r_{\sigma} \rho^{2}\right)\right. \\
& \left.-24 r_{\sigma}-4 \rho-\left(\frac{1}{2}-\lambda_{5}\right)^{2}\left(7 r_{\sigma}-34 \rho\right)\right)+\frac{320}{3} r_{\sigma}, \\
4 \pi \operatorname{Re}\left(f_{1,2}\right)= & -\frac{160}{3} r_{\sigma}
\end{aligned}
$$

\footnotetext{
${ }^{12}$ Note that in the following equations, $\lambda_{5}$ is the five-brane modulus and not the parameter belonging to the bundle data.
} 
with $\eta_{1,1}=40$ and $\eta_{2,2}=4$. The resulting gauge coupling (67) for the $U(1)_{X}$ is then positive again:

$$
\begin{aligned}
4 \pi \operatorname{Re} f_{X, X}= & \frac{65}{16}\left(\frac{1}{3 g_{s}^{2}}\left(5 r_{\sigma}^{3}-15 r_{\sigma}^{2} \rho+15 r_{\sigma} \rho^{2}\right)-24 r_{\sigma}-4 \rho\right. \\
& \left.-\left(\frac{1}{2}-\lambda_{5}\right)^{2}\left(7 r_{\sigma}-34 \rho\right)\right)+\frac{380}{3} r_{\sigma}
\end{aligned}
$$

To summarize, this example with three chiral generations satisfies the tadpole condition (61) as well as the constraints (64) guaranteeing a massless $U(1)_{X}$. We have no non-MSSM like selection rules for the Yukawa couplings of the righthanded electrons since there are indeed no contributions from the second $E_{8}(65)$. Furthermore, the Kähler moduli can be chosen such that the DUY equation (66) holds and the gauge couplings are positive.

In Appendix B, we list all three-generation models we have found on $\mathrm{dP}_{4}$ by a computer search which likewise satisfy all these conditions. We have also found three-generation examples for a scenario directly giving rise to the Standard Model gauge symmetry, to be discussed in the next section.

\section{Just the $S U(3) \times S U(2) \times U(1)_{Y}$ gauge symmetry}

Since the Standard Model contains an abelian gauge symmetry, one can try to get the Standard Model gauge symmetry directly from embedding abelian bundles into $E_{8} \times E_{8}$. The philosophy is actually very similar to the one presented in section 3 .

\section{1 $S U(5) \times U(1)$ bundles}

The direct breaking of $E_{8}$ to the Standard Model group is indeed possible by choosing a bundle with structure group $S U(5) \times U(1)$. Now we start with a bundle

$$
W=V \oplus L^{-1}, \quad \text { with } c_{1}(V)=c_{1}(L), \operatorname{rank}(V)=5,
$$

which has structure group $S U(5) \times U(1)$. This bundle $W$ can be embedded into an $S U(6)$ subgroup of $E_{8}$ such that the commutant is $S U(3) \times S U(2) \times U(1)_{1}$. We embed the $U(1)$ bundle such that

$$
\mathcal{Q}_{1}=(1,1,1,1,1,-5) .
$$

The decomposition of the adjoint representation of $E_{8}$ reads

$$
248 \stackrel{S U(5) \times S U(3) \times S U(2) \times U(1)_{1}}{\longrightarrow}\left\{\begin{array}{c}
(\mathbf{2} 4 ; \mathbf{1}, \mathbf{1})_{0}+(\mathbf{1} ; \mathbf{1}, \mathbf{1})_{0}+(\mathbf{1} ; \mathbf{8}, \mathbf{1})_{0}+(\mathbf{1} ; \mathbf{1}, \mathbf{3})_{0} \\
(\mathbf{5} ; \mathbf{3}, \mathbf{2})_{1}+(\mathbf{1} ; \mathbf{3}, \mathbf{2})_{-5}+\text { c.c. } \\
(\mathbf{1 0} ; \overline{\mathbf{3}}, \mathbf{1})_{2}+(\mathbf{5} ; \overline{\mathbf{3}}, \mathbf{1})_{-4}+\text { c.c. } \\
(\overline{\mathbf{1 0}} ; \mathbf{1}, \mathbf{2})_{3}+(\mathbf{5} ; \mathbf{1}, \mathbf{1})_{6}+\text { c.c. }
\end{array}\right\}
$$


and apparently contains states with just the Standard Model quantum numbers. Here again the $U(1)_{1}$ by itself cannot remain massless so that we will perform the same construction as for the flipped $S U(5)$ model. We embed the line bundle also in the second $E_{8}$ and realize that here the linear combination

$$
U(1)_{Y}=\frac{1}{3}\left(U(1)_{1}+3 U(1)_{2}\right)
$$

remains massless if again the conditions

$$
\int_{X} c_{1}(L) \wedge c_{2}(V)=0, \quad \int_{\Gamma_{a}} c_{1}(L)=0
$$

are satisfied. The resulting chiral massless spectrum takes the simple form given in Table 4.

\begin{tabular}{|c||c|c|}
\hline \hline$S U(3) \times S U(2) \times U(1)_{Y} \times E_{7}$ & bundle & SM part. \\
\hline \hline$(\mathbf{3}, \mathbf{2}, \mathbf{1})_{\frac{1}{3}}$ & $\chi(V)=g$ & $q_{L}$ \\
$(\mathbf{3}, \mathbf{2}, \mathbf{1})_{-\frac{5}{3}}$ & $\chi\left(L^{-1}\right)=0$ & - \\
\hline$(\overline{\mathbf{3}}, \mathbf{1}, \mathbf{1})_{\frac{2}{3}}$ & $\chi\left(\bigwedge^{2} V\right)=g$ & $d_{R}^{c}$ \\
$(\overline{\mathbf{3}}, \mathbf{1}, \mathbf{1})_{-\frac{4}{3}}$ & $\chi\left(V \otimes L^{-1}\right)=g$ & $u_{R}^{c}$ \\
\hline$(\mathbf{1}, \mathbf{2}, \mathbf{1})_{-1}$ & $\chi\left(\bigwedge^{2} V \otimes L^{-1}\right)=g$ & $l_{L}$ \\
$(\mathbf{1}, \mathbf{1}, \mathbf{1})_{2}$ & $\chi(V \otimes L)+\chi\left(L^{-2}\right)=g$ & $e_{R}^{c}$ \\
$(\mathbf{1}, \mathbf{1}, \mathbf{5 6})_{1}$ & $\chi\left(L^{-1}\right)=0$ & - \\
\hline
\end{tabular}

Table 4: Massless spectrum of $H=S U(3) \times S U(2) \times U(1)_{Y}$ models with $g=$ $\frac{1}{2} \int_{X} c_{3}(V)$.

Therefore, one gets precisely $g$ generations of Standard Model matter without a right-handed neutrino. The right-handed electrons have contributions from both the first and the second $E_{8}$. The latter are again absent if additionally one requires

$$
\int_{X} c_{1}^{3}(L)=0
$$

In this model, there are no additional gauge or obvious discrete symmetries carried by the Standard Model particles, so that the dangerous dimension four proton decay operators are not necessarily vanishing. We refer to Table 6 in Appendix $\mathrm{B}$ for a couple of three-generation examples we have found in this setup. 


\subsection{Gauge coupling unification}

Let us discuss the issue of gauge coupling unification for these models in more detail. The discussion for the flipped $S U(5)$ models is very similar. Recall that if one breaks a stringy $S U(5)$ or $S O(10)$ GUT model via discrete Wilson lines, the Standard Model gauge couplings unify at the GUT scale $2 \cdot 10^{16} \mathrm{GeV}$, i.e. at this scale they satisfy the relation $\alpha_{3}=\alpha_{2}=\frac{5}{3} \alpha_{Y}=\alpha_{G U T}$. For the weakly coupled heterotic string, however, the Planck scale comes out too low, which can be remedied in the strong coupling Horava-Witten theory [34,44,45]. Here it turned out that for the resulting values of $M_{11}, \rho$ and $r_{C Y}=M_{G U T}^{-1}$, the higher order corrections to the gauge couplings could just be ignored compared to the leading order contributions.

In our models we expect a completely different picture, as the final $U(1)_{Y}$ gauge symmetry has its origin in both $E_{8}$ walls. For the non-abelian gauge couplings of the $S U(3)$ and $S U(2)$ factors including the one-loop contribution we get

$$
\begin{aligned}
\frac{1}{\alpha_{3,2}}= & \frac{1}{3 \ell_{s}^{6} g_{s}^{2}} \int_{X} J \wedge J \wedge J-\frac{1}{\ell_{s}^{2}} \int_{X} J \wedge\left[-c_{2}(V)+c_{1}^{2}(L)+\frac{1}{2} c_{2}(T)\right] \\
& +\frac{1}{\ell_{s}^{2}} \sum_{a} N_{a}\left(\frac{1}{2}-\lambda_{a}\right)^{2} \int_{\Gamma_{a}} J
\end{aligned}
$$

Using

$$
\eta_{1,1}=60, \quad \eta_{2,2}=4, \quad \kappa_{1,1}=12, \quad \kappa_{2,2}=-4,
$$

for the abelian gauge coupling we eventually obtain

$$
\frac{1}{\alpha_{Y}}=\frac{8}{3} \frac{1}{\alpha_{3,2}}-\frac{1}{\ell_{s}^{2}} \int_{X} J \wedge\left[c_{2}(V)+4 c_{1}^{2}(L)\right]+\frac{2}{\ell_{s}^{2}} \sum_{a} N_{a} \lambda_{a} \int_{\Gamma_{a}} J .
$$

Note that these string models do not give rise to the usual GUT tree level relation $\alpha_{G U T}=\frac{5}{3} \alpha_{Y}$ but instead $\alpha_{G U T}=\frac{8}{3} \alpha_{Y}$, and therefore the tree level gauge couplings do not unify at $M_{G U T}$ (assuming just the MSSM matter content). This is the phenomenological prize we have to pay for breaking the gauge symmetry by non-trivial abelian bundles. After performing the same steps, mutatis mutandis, for our flipped $S U(5)$, we find exactly the same relation (122).

It is striking that just the gauge factor which does not unify with the other two does receive extra one-loop threshold corrections, so that in principle these can help to eventually give a unified picture. Defining

$$
\frac{1}{\alpha_{Y}}=\frac{8}{3} \frac{1}{\alpha_{G U T}}+\Delta
$$


we see that the threshold correction must take the value $\Delta=-\frac{1}{\alpha_{G U T}} \sim-24$ meaning that

$$
\left.\frac{1}{\alpha_{Y}}\right|_{1-\text { loop }}=-\left.\frac{3}{8} \frac{1}{\alpha_{Y}}\right|_{\text {tree }}
$$

For $\alpha_{G U T}=1 / 24$, such a relation can just be satisfied with $g_{s}<1$ and $r_{C Y}>\sqrt{\alpha^{\prime}}$ for large enough Chern classes of the vector bundles. Of course, in the weakly coupled heterotic framework, the Planck scale still comes out too low and one should better consider Horava-Witten theory, where now the next-to-leading order corrections to the gauge couplings should be taken into account.

Alternatively, one can contemplate that extra light Higgs fields, if present in the non-chiral spectrum, might lead to gauge coupling unification at a different scale. However, this scale is necessarily lower than the usual GUT scale, which introduces problems with fast proton decay and worsens the mismatch of the Planck scale. For instance, with three Higgs fields the three gauge couplings unify at $M_{G U T}=3 \cdot 10^{11} \mathrm{GeV}$ with a small threshold correction of only $\Delta \sim 2$.

For the example given in section 4 and setting all $\lambda_{a}=0$ for simplicity, the threshold correction is

$$
\Delta=-\frac{1}{\ell_{s}^{2}} \int_{X} J \wedge\left[c_{2}(V)+4 c_{1}^{2}(L)\right]=183 r_{\sigma}-26 \rho
$$

and has the correct sign if $r_{\sigma}<\frac{26}{183} \rho$. Note that with this choice for $r_{\sigma}$, the positivity of the gauge couplings can still be achieved.

\section{Conclusions}

The use of non-trivial line bundles considerably enlarges the model building flexibility in heterotic string compactifications. It is the aim of this publication to exemplify this by explicitly constructing realistic GUT and MSSM vacua from simply-connected Calabi-Yau manifolds carrying specific types of $U(N)$ bundles. In trying to avoid the use of discrete Wilson lines for GUT breaking we have been led to the flipped $S U(5)$ scenario, which naturally arises after embedding an $S U(4) \times U(1)$ bundle into one $E_{8}$ and a particular line bundle into the second. The GUT breaking down to $S U(3) \times S U(2) \times U(1)_{Y}$ can be further accomplished by a Higgs field in the $\mathbf{1 0}-\overline{\mathbf{1 0}}$. If one employs instead a bundle of structure group $S U(5) \times U(1)$, one can directly achieve the MSSM gauge group. The bundle data have to satisfy a couple of constraints which ensure that the hypercharge is indeed massless and that no exotic matter is present. By analysing the resulting equations on an elliptically fibered Calabi-Yau over $\mathrm{dP}_{4}$, we have given a couple of three-generation solutions to these constraints, together with the one imposed by tadpole cancellation and the loop-corrected DUY equation. 
So far, we have only explored the simplest possible choice of bundles in the hidden sector. More refined constructions of this type are conceivable, where the $U(1)_{Y}$, being a linear combination of abelian factors from both $E_{8}$ 's, might couple the MSSM matter to the (no more) hidden sector matter. This coupling would therefore be communicated by the photons as the abelian gauge bosons, whose status within the MSSM gauge bosons would thus be of a very specific type. If the hidden matter sector is sufficiently heavy, this can lead to interesting, possibly even experimentally falsifiable effects within the current phenomenological bounds. It also provides the natural arena to study the effects of gauge-mediated SUSY breaking [61].

Along the way, we have seen how the presence of five-branes, required by tadpole cancellation, modifies the usual Green-Schwarz mechanism in that additional anomaly counter terms have to be present for a consistent coupling of the five-branes to the heterotic action. The existence of these terms has been confirmed by a direct M-theory computation. In particular, in the Horava-Witten limit, the abelian background bundle on the $E_{8}$ orbifold fixed plane generates an open-membrane induced D-term potential for the five-brane if it wraps an internal two-cycle whose pull-back to the ten-dimensional end of the world carries abelian gauge flux. A combination of this novel effect with other known contributions to the $\mathrm{F}$ - and D-term potential might have interesting consequences in cosmological applications (see e.g. [62] and many more articles for discussions of five-brane potentials of cosmological relevance).

We have seen explicitly that one of the two $U(1)$ factors originally present in the model becomes massive via a Stueckelberg-type axionic coupling. In this respect, the heterotic string with $U(N)$ as opposed to $S U(N)$ bundles exhibits precisely the same features which are by now well-known in Type I and Type II orientifold constructions (consult [14] for references in that case). A very remarkable consequence of these massive $U(1)$ 's has been pointed out recently in [63], where it is argued that a background value for the field strength of the Kalb-Ramond form along the three non-compact spatial dimensions leads to a kind of aether carrying the respective global charge of the broken abelian gauge group, with prospects of an alternative solution of the baryon number asymmetry problem. It would be particularly interesting to investigate the applicability of this promising scenario in concrete models also of the heterotic string with line bundles.

The analysis given in this article has only checked that our models indeed exhibit the topological properties of the three-generation flipped $S U(5)$ and MSSM respectively. It will be important work to compute the non-chiral part of the spectrum including in particular the number of GUT and electro-weak Higgses. Likewise, an analysis of $\mu$-terms and Yukawa-couplings will be indispensible in order to finally decide about the phenomenological relevance of the concrete solutions, possibly along the lines of [64-66].

The aspect which is of prime importance to us is that the explicit construc- 
tion of similar vacua is not tied to Calabi-Yau backgrounds admitting appropriate Wilson lines. Even among the quite restricted class of elliptically fibered Calabi-Yaus we have only analysed a tiny fraction of geometric and bundle parameters when searching for interesting solutions. Many more comparable vacua can probably be constructed. Whether or not they turn out to completely reproduce the observed particle physics, they do exist as consistent solutions within the vast string landscape. A detailed investigation of the topography of this solution space, e.g. in the spirit of [67] for the hidden sector of MSSM-like Gepner model orientifolds, [68-70] for a class of toroidal intersecting braneworlds and recently [71] for the free-fermionic corner of heterotic string theory, might be one more modest step towards investigating the status of Standard Model like vacua within the landscape. After all, the goal is none less than to understand what a funny world we live in from the point of view of M-theory.

\section{Acknowledgements}

We gratefully acknowledge helpful discussions with Florian Gmeiner, Gabriele Honecker and Dimitrios Tsimpis and stimulating correspondence with Radu Tatar and Taizan Watari. We are indepted to Gabriele Honecker for many valuable comments on the manuscript.

\section{A Normalization of the Green-Schwarz term from Type I string theory}

Since there exists some confusion in the literature (including our own papers) about the correct normalization of the one-loop Green-Schwarz term

$$
S_{G S}=c \int B_{2} \wedge X_{8}
$$

for the heterotic string and Horava-Witten theory, we will now thoroughly derive this term in the S-dual Type I theory. There is overall agreement that the constant $c$ is of the form

$$
c=\frac{1}{3 \hat{c}(2 \pi)^{5} \alpha^{\prime}},
$$

but for the parameter $\hat{c}$ we found various values $\hat{c}=1,8,16$.

Let us now present our derivation from the S-dual Type IIB orientifold point of view. The starting point is the well established Type IIB action including the 
Chern-Simons terms of a stack of $M$ D9-branes,

$$
\begin{gathered}
S_{I I B}=\frac{1}{2 \kappa_{10}^{2}} \int e^{-2 \phi} R-\frac{1}{4 \kappa_{10}^{2}} \int G_{3} \wedge \star G_{3}-\frac{1}{2 g_{Y}^{2}} \int e^{-\phi} \operatorname{tr}_{U(M)}[F \wedge \star F]+ \\
\mu_{9} \int \sum_{n} C_{2 n+2} \wedge \operatorname{ch}(i \mathcal{F}) \wedge \sqrt{\hat{A}}
\end{gathered}
$$

where $\kappa_{10}^{2}=\frac{1}{2}(2 \pi)^{7}\left(\alpha^{\prime}\right)^{4}, \mu_{9}=\frac{1}{(2 \pi)^{9}\left(\alpha^{\prime}\right)^{5}}, \frac{1}{g_{Y}^{2}}=\left(2 \pi \alpha^{\prime}\right)^{2} \mu_{9}$ and the Chern characters $\operatorname{ch}_{k}(i \mathcal{F})=\frac{\ell_{s}^{2 k}}{k !(2 \pi)^{k}} \operatorname{tr}_{U(M)} F$. The traces are over the fundamental representation of the $U(M)$ gauge theory living on the D9-branes and $G_{3}=d C_{2}$ denotes the R-R three-form field strength.

Now we are taking the orientifold projection which means that we devide the entire action by a factor of two and introduce the $\Omega$ image of the stack of branes, which is a stack of $M$ D9-branes with gauge field $-F$. Tadpole cancellation fixes $M=16$. The Type I action becomes

$$
\begin{aligned}
S_{I}= & \frac{1}{4 \kappa_{10}^{2}} \int e^{-2 \phi} R-\frac{1}{8 \kappa_{10}^{2}} \int G_{3} \wedge \star G_{3}-\frac{1}{2 g_{Y}^{2}} \int e^{-\phi} \operatorname{tr}_{U(16)}[F \wedge \star F]+ \\
& \mu_{9} \int \sum_{n} C_{4 n+2} \wedge \operatorname{ch}(i \mathcal{F}) \wedge \sqrt{\hat{A}}
\end{aligned}
$$

Utilizing the trace identities

$$
\operatorname{tr}_{U(16)}\left[F^{2}\right]=\frac{1}{2} \operatorname{tr}_{S O(32)}\left[F^{2}\right] \quad \operatorname{tr}_{U(16)}\left[F^{4}\right]=\frac{1}{48} \operatorname{Tr}_{S O(32)}\left[F^{4}\right],
$$

with $\operatorname{Tr}_{S O(32)}$ denoting the trace in the adjoint representation, one can write the relevant Chern-Simons terms ${ }^{13}$ as

$$
\begin{aligned}
S_{I}= & \frac{1}{4 \kappa_{10}^{2}} \int e^{-2 \phi} R-\frac{1}{8 \kappa_{10}^{2}} \int G_{3} \wedge \star G_{3}-\frac{1}{4 g_{Y}^{2}} \int e^{-\phi} \operatorname{tr}_{S O(32)}[F \wedge \star F]+ \\
& \frac{2}{4 \kappa_{10}^{2}} \frac{\alpha^{\prime}}{4} \int C_{6} \wedge \operatorname{tr}_{S O(32)}\left[F^{2}\right]+\frac{1}{48(2 \pi)^{5} \alpha^{\prime}} \int C_{2} \wedge \frac{1}{24} \operatorname{Tr}_{S O(32)}\left[F^{4}\right],(131)
\end{aligned}
$$

from which one might conclude that the normalization is $\hat{c}=16$. This is however not correct as the kinetic terms are not yet canonically normalized. In order to bring all three kinetic terms to the canonical form, one has to rescale

$$
C_{2} \rightarrow 2 \sqrt{2} C_{2}, \quad \alpha^{\prime} \rightarrow \sqrt{2} \alpha^{\prime}, \quad e^{\phi} \rightarrow \frac{1}{2 \sqrt{2}} e^{\phi} .
$$

This leads to the action

$$
\begin{aligned}
S_{I}= & \frac{1}{2 \kappa_{10}^{2}} \int e^{-2 \phi} R-\frac{1}{4 \kappa_{10}^{2}} \int G_{3} \wedge \star G_{3}-\frac{1}{2 \bar{g}_{Y}^{2}} \int e^{-\phi} \operatorname{tr}_{S O(32)}[F \wedge \star F]+ \\
& \frac{2}{4 \kappa_{10}^{2}} \frac{\alpha^{\prime}}{4} \int C_{6} \wedge \operatorname{tr}_{S O(32)}\left[F^{2}\right]+\frac{1}{24(2 \pi)^{5} \alpha^{\prime}} \int C_{2} \wedge \frac{1}{24} \operatorname{Tr}_{S O(32)}\left[F^{4}\right]
\end{aligned}
$$

\footnotetext{
${ }^{13}$ Since we are only interested in the normalization factors, we just consider the F-dependent pieces and do not explicitly write down the curvature pieces arising from the $\hat{A}$-genus.
} 
with the Type I gauge coupling $\frac{1}{\bar{g}_{Y}^{2}}=\frac{1}{2(2 \pi)^{7}\left(\alpha^{\prime}\right)^{3}}$. This action is really S-dual to the heterotic string action as given e.g. in [38]. Therefore we conclude that the correct normalization of the heterotic Green-Schwarz term is $\hat{c}=8$, so that

$$
S_{G S}=\frac{1}{24(2 \pi)^{5} \alpha^{\prime}} \int B_{2} \wedge X_{8}
$$

if the all the fields are canonically normalized as in [38].

\section{B Three-generation examples}

We list all examples we have found by a computer search on elliptically fibered Calabi-Yau spaces with base spaces $\mathrm{dP}_{r}, r=1, \ldots, 4$ and the Hirzebruch surfaces $F_{r}$ in a range from $-10, \ldots, 10$ for all parameters. We have found three-generation models only on $\mathrm{dP}_{4}$. Table 5 contains the three-generation flipped $S U(5)$ models, whereas in table 6 we list all three-generation vacua directly with MSSM gauge group (see section 5) which we have found.

\begin{tabular}{|c||c|c|c|c|}
\hline \hline$\lambda$ & $\eta$ & $q$ & $c_{1}(\zeta)$ & {$[W]$} \\
\hline \hline$\frac{1}{4}$ & $14 l-2 E_{1}-6 E_{2}-6 E_{3}-2 E_{4}$ & 0 & $-l+E_{2}+E_{3}+E_{4}$ & $27 F+\left(22 l-10 E_{1}-6 E_{2}-6 E_{3}-10 E_{4}\right) \sigma$ \\
\hline$\frac{1}{4}$ & $18 l-10 E_{1}-6 E_{2}-6 E_{3}-6 E_{4}$ & 0 & $-l+E_{2}+E_{3}+E_{4}$ & $27 F+\left(18 l-2 E_{1}-6 E_{2}-6 E_{3}-6 E_{4}\right) \sigma$ \\
\hline$\frac{1}{4}$ & $14 l-6 E_{1}-2 E_{2}-2 E_{3}-6 E_{4}$ & 0 & $-E_{1}+E_{4}$ & $27 F+\left(22 l-6 E_{1}-10 E_{2}-10 E_{3}-6 E_{4}\right) \sigma$ \\
\hline$\frac{1}{4}$ & $14 l-2 E_{1}-6 E_{2}-6 E_{3}-2 E_{4}$ & 0 & $-E_{1}+E_{4}$ & $27 F+\left(22 l-10 E_{1}-6 E_{2}-6 E_{3}-10 E_{4}\right) \sigma$ \\
\hline$\frac{1}{4}$ & $18 l-6 E_{1}-10 E_{2}-6 E_{3}-6 E_{4}$ & 0 & $-E_{1}+E_{4}$ & $27 F+\left(18 l-6 E_{1}-2 E_{2}-6 E_{3}-6 E_{4}\right) \sigma$ \\
\hline$\frac{1}{4}$ & $14 l-2 E_{1}-6 E_{2}-6 E_{3}-2 E_{4}$ & 0 & $l-E_{1}-E_{2}-E_{3}$ & $27 F+\left(22 l-10 E_{1}-6 E_{2}-6 E_{3}-10 E_{4}\right) \sigma$ \\
\hline$\frac{1}{4}$ & $18 l-6 E_{1}-6 E_{2}-6 E_{3}-10 E_{4}$ & 0 & $l-E_{1}-E_{2}-E_{3}$ & $27 F+\left(18 l-6 E_{1}-6 E_{2}-6 E_{3}-2 E_{4}\right) \sigma$ \\
\hline
\end{tabular}

Table 5: Flipped $S U(5) \times U(1)_{X}$ models on $\mathrm{dP}_{4}$. 


\begin{tabular}{|c|c|c|c|c|}
\hline$\lambda$ & $\eta$ & $q$ & $c_{1}(\zeta)$ & {$[W]$} \\
\hline$\frac{1}{2}$ & $15 l-3 E_{1}-5 E_{2}-5 E_{3}-5 E_{4}$ & 0 & $-l+E_{2}+E_{3}+E_{4}$ & $7 F+\left(21 l-9 E_{1}-7 E_{2}-7 E_{3}-7 E_{4}\right) \sigma$ \\
\hline$\frac{1}{2}$ & $15 l-2 E_{1}-5 E_{2}-5 E_{3}-5 E_{4}$ & 0 & $-l+E_{2}+E_{3}+E_{4}$ & $7 F+\left(21 l-10 E_{1}-7 E_{2}-7 E_{3}-7 E_{4}\right) \sigma$ \\
\hline$\frac{1}{2}$ & $17 l-7 E_{1}-7 E_{2}-5 E_{3}-5 E_{4}$ & 0 & $-l+E_{2}+E_{3}+E_{4}$ & $7 F+\left(19 l-5 E_{1}-5 E_{2}-7 E_{3}-7 E_{4}\right) \sigma$ \\
\hline$\frac{1}{2}$ & $18 l-8 E_{1}-8 E_{2}-5 E_{3}-5 E_{4}$ & 0 & $-l+E_{2}+E_{3}+E_{4}$ & $7 F+\left(18 l-4 E_{1}-4 E_{2}-7 E_{3}-7 E_{4}\right) \sigma$ \\
\hline$\frac{1}{2}$ & $20 l-3 E_{1}-10 E_{2}-10 E_{3}$ & 0 & $-l+E_{2}+E_{3}+E_{4}$ & $7 F+\left(16 l-9 E_{1}-2 E_{2}-2 E_{3}-12 E_{4}\right) \sigma$ \\
\hline$\frac{1}{2}$ & $20 l-2 E_{1}-10 E_{2}-10 E_{3}$ & 0 & $-l+E_{2}+E_{3}+E_{4}$ & $7 F+\left(16 l-10 E_{1}-2 E_{2}-2 E_{3}-12 E_{4}\right) \sigma$ \\
\hline$\frac{1}{2}$ & $15 l-5 E_{1}-5 E_{2}-3 E_{3}-5 E_{4}$ & 0 & $-E_{1}+E_{4}$ & $7 F+\left(21 l-7 E_{1}-7 E_{2}-9 E_{3}-7 E_{4}\right) \sigma$ \\
\hline$\frac{1}{2}$ & $15 l-5 E_{1}-5 E_{2}-2 E_{3}-5 E_{4}$ & 0 & $-E_{1}+E_{4}$ & $7 F+\left(21 l-7 E_{1}-7 E_{2}-10 E_{3}-7 E_{4}\right) \sigma$ \\
\hline$\frac{1}{2}$ & $15 l-5 E_{1}-3 E_{2}-5 E_{4}$ & 0 & $-E_{1}+E_{4}$ & $7 F+\left(21 l-7 E_{1}-9 E_{2}-12 E_{3}-7 E_{4}\right) \sigma$ \\
\hline$\frac{1}{2}$ & $15 l-5 E_{1}-2 E_{2}-5 E_{4}$ & 0 & $-E_{1}+E_{4}$ & $7 F+\left(21 l-7 E_{1}-10 E_{2}-12 E_{3}-7 E_{4}\right) \sigma$ \\
\hline$\frac{1}{2}$ & $15 l-5 E_{2}-3 E_{3}$ & 0 & $-E_{1}+E_{4}$ & $7 F+\left(21 l-12 E_{1}-7 E_{2}-9 E_{3}-12 E_{4}\right) \sigma$ \\
\hline$\frac{1}{2}$ & $17 l-7 E_{1}-5 E_{2}-5 E_{3}-7 E_{4}$ & 0 & $-E_{1}+E_{4}$ & $7 F+\left(19 l-5 E_{1}-7 E_{2}-7 E_{3}-5 E_{4}\right) \sigma$ \\
\hline$\frac{1}{2}$ & $17 l-7 E_{1}-5 E_{2}-7 E_{4}$ & 0 & $-E_{1}+E_{4}$ & $7 F+\left(19 l-5 E_{1}-7 E_{2}-12 E_{3}-5 E_{4}\right) \sigma$ \\
\hline$\frac{1}{2}$ & $17 l-7 E_{1}-7 E_{4}$ & 0 & $-E_{1}+E_{4}$ & $7 F+\left(19 l-5 E_{1}-12 E_{2}-12 E_{3}-5 E_{4}\right) \sigma$ \\
\hline$\frac{1}{2}$ & $17 l-5 E_{1}-7 E_{2}-7 E_{3}-5 E_{4}$ & 0 & $-E_{1}+E_{4}$ & $7 F+\left(19 l-7 E_{1}-5 E_{2}-5 E_{3}-7 E_{4}\right) \sigma$ \\
\hline$\frac{1}{2}$ & $17 l-7 E_{2}-7 E_{3}$ & 0 & $-E_{1}+E_{4}$ & $7 F+\left(19 l-12 E_{1}-5 E_{2}-5 E_{3}-12 E_{4}\right) \sigma$ \\
\hline$\frac{1}{2}$ & $18 l-8 E_{1}-5 E_{2}-5 E_{3}-8 E_{4}$ & 0 & $-E_{1}+E_{4}$ & $7 F+\left(18 l-4 E_{1}-7 E_{2}-7 E_{3}-4 E_{4}\right) \sigma$ \\
\hline$\frac{1}{2}$ & $18 l-8 E_{1}-5 E_{2}-8 E_{4}$ & 0 & $-E_{1}+E_{4}$ & $7 F+\left(18 l-4 E_{1}-7 E_{2}-12 E_{3}-4 E_{4}\right) \sigma$ \\
\hline$\frac{1}{2}$ & $18 l-8 E_{1}-8 E_{4}$ & 0 & $-E_{1}+E_{4}$ & $7 F+\left(18 l-4 E_{1}-12 E_{2}-12 E_{3}-4 E_{4}\right) \sigma$ \\
\hline$\frac{1}{2}$ & $18 l-5 E_{1}-8 E_{2}-8 E_{3}-5 E_{4}$ & 0 & $-E_{1}+E_{4}$ & $7 F+\left(18 l-7 E_{1}-4 E_{2}-4 E_{3}-7 E_{4}\right) \sigma$ \\
\hline$\frac{1}{2}$ & $18 l-8 E_{2}-8 E_{3}$ & 0 & $-E_{1}+E_{4}$ & $7 F+\left(18 l-12 E_{1}-4 E_{2}-4 E_{3}-12 E_{4}\right) \sigma$ \\
\hline$\frac{1}{2}$ & $20 l-10 E_{1}-5 E_{2}-3 E_{3}-10 E_{4}$ & 0 & $-E_{1}+E_{4}$ & $7 F+\left(16 l-2 E_{1}-7 E_{2}-9 E_{3}-2 E_{4}\right) \sigma$ \\
\hline$\frac{1}{2}$ & $20 l-10 E_{1}-5 E_{2}-2 E_{3}-10 E_{4}$ & 0 & $-E_{1}+E_{4}$ & $7 F+\left(16 l-2 E_{1}-7 E_{2}-10 E_{3}-2 E_{4}\right) \sigma$ \\
\hline$\frac{1}{2}$ & $20 l-10 E_{1}-3 E_{2}-10 E_{4}$ & 0 & $-E_{1}+E_{4}$ & $7 F+\left(16 l-2 E_{1}-9 E_{2}-12 E_{3}-2 E_{4}\right) \sigma$ \\
\hline$\frac{1}{2}$ & $20 l-10 E_{1}-2 E_{2}-10 E_{4}$ & 0 & $-E_{1}+E_{4}$ & $7 F+\left(16 l-2 E_{1}-10 E_{2}-12 E_{3}-2 E_{4}\right) \sigma$ \\
\hline$\frac{1}{2}$ & $15 l-5 E_{1}-5 E_{2}-5 E_{3}-3 E_{4}$ & 0 & $l-E_{1}-E_{2}-E_{3}$ & $7 F+\left(21 l-7 E_{1}-7 E_{2}-7 E_{3}-9 E_{4}\right) \sigma$ \\
\hline$\frac{1}{2}$ & $15 l-5 E_{1}-5 E_{2}-5 E_{3}-2 E_{4}$ & 0 & $l-E_{1}-E_{2}-E_{3}$ & $7 F+\left(21 l-7 E_{1}-7 E_{2}-7 E_{3}-10 E_{4}\right) \sigma$ \\
\hline$\frac{1}{2}$ & $17 l-7 E_{1}-5 E_{2}-5 E_{3}-7 E_{4}$ & 0 & $l-E_{1}-E_{2}-E_{3}$ & $7 F+\left(19 l-5 E_{1}-7 E_{2}-7 E_{3}-5 E_{4}\right) \sigma$ \\
\hline$\frac{1}{2}$ & $18 l-8 E_{1}-5 E_{2}-5 E_{3}-8 E_{4}$ & 0 & $l-E_{1}-E_{2}-E_{3}$ & $7 F+\left(18 l-4 E_{1}-7 E_{2}-7 E_{3}-4 E_{4}\right) \sigma$ \\
\hline$\frac{1}{2}$ & $20 l-10 E_{1}-10 E_{2}-3 E_{4}$ & 0 & $l-E_{1}-E_{2}-E_{3}$ & $7 F+\left(16 l-2 E_{1}-2 E_{2}-12 E_{3}-9 E_{4}\right) \sigma$ \\
\hline$\frac{1}{2}$ & $20 l-10 E_{1}-10 E_{2}-2 E_{4}$ & 0 & $l-E_{1}-E_{2}-E_{3}$ & $7 F+\left(16 l-2 E_{1}-2 E_{2}-12 E_{3}-10 E_{4}\right) \sigma$ \\
\hline
\end{tabular}

Table 6: $S U(3) \times S U(2) \times U(1)$ models on $\mathrm{dP}_{4}$. 


\section{References}

[1] E. Witten, "New issues in manifolds of su(3) holonomy," Nucl. Phys. B268 (1986) 79 .

[2] R. Donagi, B. A. Ovrut, T. Pantev, and D. Waldram, "Standard-model bundles on non-simply connected calabi-yau threefolds," JHEP 08 (2001) 053, hep-th/0008008.

[3] R. Donagi, B. A. Ovrut, T. Pantev, and D. Waldram, "Standard-model bundles," Adv. Theor. Math. Phys. 5 (2002) 563-615, math.ag/0008010.

[4] R. Donagi, B. A. Ovrut, T. Pantev, and D. Waldram, "Spectral involutions on rational elliptic surfaces," Adv. Theor. Math. Phys. 5 (2002) 499-561, math.ag/0008011.

[5] V. Braun, B. A. Ovrut, T. Pantev, and R. Reinbacher, "Elliptic calabi-yau threefolds with z(3) x z(3) wilson lines," JHEP 12 (2004) 062, hep-th/0410055.

[6] B. Andreas, G. Curio, and A. Klemm, "Towards the standard model spectrum from elliptic calabi- yau," Int. J. Mod. Phys. A19 (2004) 1987, hep-th/9903052.

[7] G. Curio, "Standard model bundles of the heterotic string," hep-th/0412182.

[8] V. Bouchard and R. Donagi, "An su(5) heterotic standard model," Phys. Lett. B633 (2006) 783-791, hep-th/0512149.

[9] V. Braun, Y.-H. He, B. A. Ovrut, and T. Pantev, "A heterotic standard model," Phys. Lett. B618 (2005) 252-258, hep-th/0501070.

[10] V. Braun, Y.-H. He, B. A. Ovrut, and T. Pantev, "The exact mssm spectrum from string theory," hep-th/0512177.

[11] T. L. Gomez, S. Lukic, and I. Sols, "Constraining the kaehler moduli in the heterotic standard model," hep-th/0512205.

[12] V. Braun, Y.-H. He, and B. A. Ovrut, "Stability of the minimal heterotic standard model bundle," hep-th/0602073.

[13] W. Buchmuller, K. Hamaguchi, O. Lebedev, and M. Ratz, "The supersymmetric standard model from the heterotic string," hep-ph/0511035.

[14] R. Blumenhagen, M. Cvetic, P. Langacker, and G. Shiu, "Toward realistic intersecting d-brane models," hep-th/0502005. 
[15] M. B. Green, J. H. Schwarz, and P. C. West, "Anomaly free chiral theories in six dimensions," Nucl. Phys. B254 (1985) 327-348.

[16] J. Distler and B. R. Greene, "Aspects of $(2,0)$ string compactifications," Nucl. Phys. B304 (1988) 1.

[17] G. Aldazabal, A. Font, L. E. Ibanez, and A. M. Uranga, "New branches of string compactifications and their f-theory duals," Nucl. Phys. B492 (1997) 119-151, hep-th/9607121.

[18] A. Lukas and K. S. Stelle, "Heterotic anomaly cancellation in five dimensions," JHEP 01 (2000) 010, hep-th/9911156.

[19] B. Andreas and D. Hernandez Ruiperez, "U(n) vector bundles on calabi-yau threefolds for string theory compactifications," hep-th/0410170

[20] R. Blumenhagen, G. Honecker, and T. Weigand, "Supersymmetric (non-)abelian bundles in the type i and so(32) heterotic string," JHEP 08 (2005) 009, hep-th/0507041.

[21] R. Blumenhagen, G. Honecker, and T. Weigand, "Non-abelian brane worlds: The heterotic string story," JHEP 10 (2005) 086, hep-th/0510049

[22] R. Blumenhagen, G. Honecker, and T. Weigand, "Non-abelian brane worlds: The open string story," hep-th/0510050.

[23] R. Blumenhagen, G. Honecker, and T. Weigand, "Loop-corrected compactifications of the heterotic string with line bundles," JHEP 06 (2005) 020, hep-th/0504232.

[24] S. M. Barr, "A new symmetry breaking pattern for so(10) and proton decay," Phys. Lett. B112 (1982) 219.

[25] J. P. Derendinger, J. E. Kim, and D. V. Nanopoulos, "Anti - su(5)," Phys. Lett. B139 (1984) 170.

[26] I. Antoniadis, J. R. Ellis, J. S. Hagelin, and D. V. Nanopoulos, "The flipped su(5) x u(1) string model revamped," Phys. Lett. B231 (1989) 65.

[27] C.-M. Chen, V. E. Mayes, and D. V. Nanopoulos, "Flipped su(5) from d-branes with type iib fluxes," Phys. Lett. B633 (2006) 618-626, hep-th/0511135.

[28] R. Friedman, J. W. Morgan, and E. Witten, "Vector bundles over elliptic fibrations," alg-geom/9709029.

[29] R. Friedman, J. Morgan, and E. Witten, "Vector bundles and f theory," Commun. Math. Phys. 187 (1997) 679-743, hep-th/9701162. 
[30] G. Honecker, "Massive u(1)s and heterotic five-branes on k3," hep-th/0602101.

[31] A. Lukas, B. A. Ovrut, and D. Waldram, "On the four-dimensional effective action of strongly coupled heterotic string theory," Nucl. Phys. B532 (1998) 43-82, hep-th/9710208.

[32] A. Lukas, B. A. Ovrut, and D. Waldram, "Non-standard embedding and five-branes in heterotic m- theory," Phys. Rev. D59 (1999) 106005, hep-th/9808101.

[33] I. Antoniadis, J. R. Ellis, J. S. Hagelin, and D. V. Nanopoulos, "Supersymmetric flipped su(5) revitalized," Phys. Lett. B194 (1987) 231.

[34] E. Witten, "Strong coupling expansion of calabi-yau compactification," Nucl. Phys. B471 (1996) 135-158, hep-th/9602070.

[35] R. Tatar and T. Watari, "Proton decay, yukawa couplings and underlying gauge symmetry in string theory," hep-th/0602238.

[36] B. Andreas and G. Curio, "Standard models from heterotic string theory," hep-th/0602247.

[37] T. Weigand, "Heterotic vacua from general (non-) abelian bundles," hep-th/0512191.

[38] J. Polchinski, "String theory. vol. 2: Superstring theory and beyond,". Cambridge, UK: Univ. Pr. (1998) 531 p.

[39] M. B. Green and J. H. Schwarz, "Anomaly cancellation in supersymmetric d=10 gauge theory and superstring theory," Phys. Lett. B149 (1984) $117-122$.

[40] L. E. Ibanez and H. P. Nilles, "Low-energy remnants of superstring anomaly cancellation terms," Phys. Lett. B169 (1986) 354.

[41] P. Pasti, D. P. Sorokin, and M. Tonin, "Covariant action for a d = 11 five-brane with the chiral field," Phys. Lett. B398 (1997) 41-46, hep-th/9701037.

[42] A. Lukas, B. A. Ovrut, and D. Waldram, "The ten-dimensional effective action of strongly coupled heterotic string theory," Nucl. Phys. B540 (1999) 230-246, hep-th/9801087.

[43] L. Carlevaro and J.-P. Derendinger, "Five-brane thresholds and membrane instantons in four- dimensional heterotic m-theory," Nucl. Phys. B736 (2006) 1-33, hep-th/0502225. 
[44] P. Horava and E. Witten, "Heterotic and type i string dynamics from eleven dimensions," Nucl. Phys. B460 (1996) 506-524, hep-th/9510209.

[45] P. Horava and E. Witten, "Eleven-dimensional supergravity on a manifold with boundary," Nucl. Phys. B475 (1996) 94-114, hep-th/9603142.

[46] J. O. Conrad, "Brane tensions and coupling constants from within mtheory," Phys. Lett. B421 (1998) 119-124, hep-th/9708031.

[47] M. Brandle and A. Lukas, "Five-branes in heterotic brane-world theories," Phys. Rev. D65 (2002) 064024, hep-th/0109173.

[48] J. P. Derendinger, L. E. Ibanez, and H. P. Nilles, "On the low-energy limit of superstring theories," Nucl. Phys. B267 (1986) 365.

[49] K. Choi and J. E. Kim, "Compactification and axions in e(8)xe(8) superstring models," Phys. Lett. B165 (1985) 71.

[50] H. P. Nilles and S. Stieberger, "String unification, universal one-loop corrections and strongly coupled heterotic string theory," Nucl. Phys. B499 (1997) 3-28, hep-th/9702110.

[51] S. Stieberger, "(0,2) heterotic gauge couplings and their m-theory origin," Nucl. Phys. B541 (1999) 109-144, hep-th/9807124

[52] J.-P. Derendinger and R. Sauser, "A five-brane modulus in the effective $\mathrm{n}$ = 1 supergravity of m-theory," Nucl. Phys. B598 (2001) 87-114, hep-th/0009054.

[53] G. W. Moore, G. Peradze, and N. Saulina, "Instabilities in heterotic m-theory induced by open membrane instantons," Nucl. Phys. B607 (2001) 117-154, hep-th/0012104.

[54] M. Dine, N. Seiberg, and E. Witten, "Fayet-iliopoulos terms in string theory," Nucl. Phys. B289 (1987) 589.

[55] I. Dorsner and P. Fileviez Perez, "Could we rotate proton decay away?," Phys. Lett. B606 (2005) 367-370, hep-ph/0409190

[56] P. Nath and P. F. Perez, "Proton stability in grand unified theories, in strings, and in branes," hep-ph/0601023.

[57] R. Donagi, A. Lukas, B. A. Ovrut, and D. Waldram, "Holomorphic vector bundles and non-perturbative vacua in m- theory," JHEP 06 (1999) 034, hep-th/9901009. 
[58] R. Donagi, Y.-H. He, B. A. Ovrut, and R. Reinbacher, "The particle spectrum of heterotic compactifications," JHEP 12 (2004) 054, hep-th/0405014.

[59] G. Curio, "Chiral matter and transitions in heterotic string models," Phys. Lett. B435 (1998) 39-48, hep-th/9803224.

[60] B. Andreas, "On vector bundles and chiral matter in $\mathrm{n}=1$ heterotic compactifications," JHEP 01 (1999) 011, hep-th/9802202.

[61] D.-E. Diaconescu, B. Florea, S. Kachru, and P. Svrcek, "Gauge - mediated supersymmetry breaking in string compactifications," hep-th/0512170.

[62] M. Becker, G. Curio, and A. Krause, "De sitter vacua from heterotic m-theory," Nucl. Phys. B693 (2004) 223-260, hep-th/0403027.

[63] L. E. Ibanez, "Flux-induced baryon asymmetry," hep-th/0602279.

[64] V. Braun, Y.-H. He, B. A. Ovrut, and T. Pantev, "Moduli dependent mu-terms in a heterotic standard model," hep-th/0510142.

[65] V. Braun, Y.-H. He, and B. A. Ovrut, "Yukawa couplings in heterotic standard models," hep-th/0601204.

[66] V. Bouchard, M. Cvetic, and R. Donagi, "Tri-linear couplings in an heterotic minimal supersymmetric standard model," hep-th/0602096

[67] T. P. T. Dijkstra, L. R. Huiszoon, and A. N. Schellekens, "Supersymmetric standard model spectra from rcft orientifolds," Nucl. Phys. B710 (2005) 3-57, hep-th/0411129.

[68] R. Blumenhagen, F. Gmeiner, G. Honecker, D. Lüst, and T. Weigand, "The statistics of supersymmetric d-brane models," Nucl. Phys. B713 (2005) 83-135, hep-th/0411173.

[69] F. Gmeiner, R. Blumenhagen, G. Honecker, D. Lust, and T. Weigand, "One in a billion: Mssm-like d-brane statistics," JHEP 01 (2006) 004, hep-th/0510170.

[70] F. Gmeiner, "Standard model statistics of a type ii orientifold," hep-th/0512190.

[71] K. R. Dienes, "Statistics on the heterotic landscape: Gauge groups and cosmological constants of four-dimensional heterotic strings," hep-th/0602286. 